\title{
Metal distribution in sloshing galaxy clusters: the case of A496 ${ }^{\star}$
}

\author{
Simona Ghizzardi ${ }^{1}$, Sabrina De Grandi ${ }^{2}$, and Silvano Molendi ${ }^{1}$ \\ ${ }^{1}$ INAF - Istituto di Astrofisica Spaziale e Fisica Cosmica - Milano, via E. Bassini 15, 20133 Milano, Italy \\ e-mail: simona@iasf-milano.inaf.it \\ 2 INAF - Osservatorio Astronomico di Brera, via E. Bianchi 46, 23807 Merate (LC), Italy
}

Received 16 April 2014 / Accepted 18 July 2014

ABSTRACT

\begin{abstract}
We report results from a detailed study of the sloshing gas in the core of A496. We detected the low-temperature/entropy spiral feature found in several cores. We also found that conduction between the gas in the spiral and the ambient medium must be suppressed by more than one order of magnitude compared with the Spitzer conductivity. Intriguingly, while the gas in the spiral pattern features a higher metal abundance than the surrounding medium, it follows the relation of entropy vs metal abundance defined by gas outside the spiral. The most plausible explanation for this behavior is that the low-entropy metal-rich plasma that is lifted up through the cluster atmosphere by sloshing suffers little heating or mixing with the ambient medium. While sloshing appears to be capable of lifting up significant amount of gas, the limited heat exchange and mixing between gas within and outside the spiral implies that this mechanism is not at all effective in 1) permanently redistributing metals within the core region and 2) heating up the coolest and densest gas, thereby providing little or no contribution to the staving off of catastrophic cooling in cool cores.
\end{abstract}

Key words. galaxies: clusters: intracluster medium - X-rays: galaxies: clusters - galaxies: clusters: individual: A496

\section{Introduction}

High-resolution observations have revealed a wealth of smallscale substructures within the intracluster gas of galaxy groups and clusters during the past decade, thanks to the advent of the current generation of X-ray telescopes (mainly XMM-Newton and Chandra). Particularly remarkable features are cold fronts (see Markevitch \& Vikhlinin 2007, for a detailed review). These appear as sharp surface brightness discontinuities that are accompanied by a temperature jump, where the denser gas is also cooler, so that the pressure is approximately continuous across the front. Soon after their discovery in the early Chandra observations of A2142 and A3667 (Markevitch et al. 2000; Vikhlinin et al. 2001a,b; Vikhlinin \& Markevitch 2002), these features have been often observed in many galaxy clusters. Indeed, cold fronts appear to be almost ubiquitous in galaxy clusters (Markevitch et al. 2003b; Ghizzardi et al. 2010, G10 hereafter) with the vast majority of clusters hosting at least one cold front and many cool-core clusters featuring more than one (G10). More recently, several cold fronts have also been detected in galaxy groups (NGC 5044: O'Sullivan et al. 2014; Gastaldello et al. 2009; David et al. 2009; Buote et al. 2003; NGC 5846: Machacek et al. 2011; 3C 449 Group: Lal et al. 2013; IC 1860: Gastaldello et al. 2013; Pegasus Group: Randall et al. 2009a; NGC 5098: Randall et al. 2009b). Since understanding the nature of this widespread phenomenon is mandatory for characterizing the dynamics of galaxy clusters, cold fronts have been often investigated in the past years by several authors with X-ray observations (e.g., Canning et al. 2013; Paterno-Mahler et al. 2013; Clarke et al. 2013; Rossetti et al. 2013; Ettori et al. 2013; Gastaldello et al. 2013, among the most recent works), optical data (e.g., Owers et al. 2011, 2009a,b) and numerical simulations

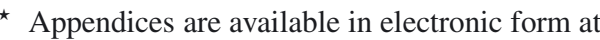
http://www . aanda.org
}

(see, e.g., Ascasibar \& Markevitch 2006; Hallman et al. 2010; ZuHone et al. 2010, 2011; Roediger \& ZuHone 2012).

In the currently dominant picture, cold fronts in cool-core clusters are believed to be caused by the sloshing of the innermost cool gas within the underlying dark matter potential well (Tittley \& Henriksen 2005; Ascasibar \& Markevitch 2006). Numerical simulations show that sloshing can be triggered within cool cores by an off-axis minor merger: an infalling subclump induces a perturbation of the underlying gravitational potential of the main cluster; the cooler inner gas is displaced from the center of the potential well, lifted up, decoupled from the dark matter through ram pressure, and finally starts to slosh. The oscillations generated by the gravitational disturbance are long-lasting, can survive for several Gyr and produce a succession of concentric fronts, that propagate outward. If the initial fly-by is off-center, the cool gas acquires angular momentum and the sloshing takes a spiral-like appearance. Simulations by Ascasibar \& Markevitch (2006) highlighted that a steep central entropy is a necessary condition for the onset of sloshing. On the observational side this requirement has been confirmed by G10 (see, however, the case of A2142, Rossetti et al. 2013).

Although they have been widely investigated, cold fronts and sloshing studies have focused mainly on dynamics and thermodynamics, while the relationship between the sloshing and the chemical properties of clusters has enjoyed relatively little attention. A detailed characterization of the metal abundance across the fronts is only available for a handful of objects (Perseus: Fabian et al. 2011; Centaurus: Sanders \& Fabian 2006; A2204: Sanders et al. 2005, 2009; A2052: de Plaa et al. 2010; A1201: Ma et al. 2012; M 87: Simionescu et al. 2010; A3581: Canning et al. 2013), and recently O'Sullivan et al. (2014) found a close correlation between the metallicity distribution and the cold-front position in the galaxy group NGC 5044. This is a significant limitation because sloshing may play a 
crucial role in redistributing metals in the intracluster medium (ICM). Notoriously, cool-core clusters have prominent metallicity peaks (De Grandi \& Molendi 2001) in their centers, consistent with being produced by the central brightest cluster galaxy (BCG; e.g., De Grandi \& Molendi 2001; De Grandi et al. 2004). As a consequence, heavy elements are powerful markers of the central cool gas and the metal distribution may trace the history of the central gas motions during the sloshing. Several works have shown that the iron abundance profile is broader than the stellar light profile of the BCG (e.g., De Grandi \& Molendi 2001; Graham et al. 2006; Rebusco et al. 2006), suggesting that metals drift away from the BGC and spread outward. The mechanism responsible for the mismatch between the metal abundance and light profiles is still poorly understood, however. Sloshing may be a viable way to broaden the distribution of the metal abundance profile.

In this paper we aim to characterize the sloshing mechanism through the metal distribution in the ICM. To address this question, we choose to analyze a long (120 ks) XMM-Newton observation of A496. A496 is a bright, nearby $(z=0.0329)$, cool-core cluster (Peres et al. 1998). It is a particularly suited candidate for inspecting the correlation between metal distribution and cold fronts because it is the only cluster to host four cold fronts (Ghizzardi et al. 2013) in its circum-core area ( $\$ 250 \mathrm{kpc})$. In addition, A496 has no other particular feature within its core (such as bubbles, rims, cavities) that would add complexity to the problem and to interpretating the results.

The paper is organized as follows: in Sect. 2 we describe the XMM observation, the data reduction, the procedure used to produce the thermodynamical maps, and the spectral extraction methods used in our analysis. In Sect. 3 we present the detection and characterization of cold fronts and other sloshing signatures, including the metal abundance distribution. We discuss results in Sect. 4 and summarize our findings in Sect. 5. If not stated otherwise, we plot and tabulate values with errors quoted at the $68 \%(1 \sigma)$ confidence level throughout the article. In our analysis, we assume a flat $\Lambda C D M$ cosmology with a Hubble constant $H_{0}=70 \mathrm{~km} \mathrm{~s}^{-1} \mathrm{Mpc}^{-1}, \Omega_{\mathrm{m}}=0.3, \Omega_{\Lambda}=0.7$. A496 is assumed to have a redshift $z=0.0329$ : at this distance 1 arcsec corresponds to $0.65 \mathrm{kpc}$. All the metallicities are given relative to the solar abundance in Anders \& Grevesse (1989).

\section{Data sets and data processing}

We used two XMM-Newton observations of Abell 496 performed on August 11, 2007 (observation id. 0506260301) and on February 18, 2008 (0506260401) for a total nominal exposure time of $141 \mathrm{ks}$. We reprocessed the observation data files (ODF) of each observation separately using the science analysis system (SAS) version 11.0.0. After producting the calibrated event lists for the EPIC MOS1, MOS2, and $p n$ data with the tasks emchain and epchain, we performed a soft-proton cleaning using a double-filtering process. We first removed soft proton spikes by screening the light curves produced in 100-s bins in the $10-12 \mathrm{keV}$ band for MOS and 10-13 keV for $p n$ (as a safety check for possible flares with soft spectra) and then by applying the appropriate threshold for each instrument: for MOS we used slightly different thresholds in the two observations, namely $0.20 \mathrm{cts} \mathrm{s}^{-1}$ for 0506260301 and $0.15 \mathrm{cts} \mathrm{s}^{-1}$ for 0506260401 , for the $p n$ instead we always used a threshold of $0.60 \mathrm{cts} \mathrm{s}^{-1}$. These thresholds are slightly higher than standard because of a higher level of soft protons. To exclude possible residual flares contributing below $10 \mathrm{keV}$, we extracted a light curve in the $2-5 \mathrm{keV}$ band and fitted the histogram produced from this curve with a Gaussian distribution. To generate the final filtered event files we rejected all events registered at times with count rates higher than $3 \sigma$ from the mean of this distribution. We finally filtered event files according to FLAG $(\mathrm{FLAG}==0)$ and PATTERN (PATTERN $\leq 12$ for MOS and PATTERN==0 for $p n$ ) criteria.

The resulting net exposure times for dataset 0506260301 are $57.1 \mathrm{ks}, 58.7 \mathrm{ks}$, and $28.3 \mathrm{ks}$ for the MOS1, MOS2, and pn detectors, respectively, while for the 0506260401 observations the exposures are $53.0 \mathrm{ks}, 47.8 \mathrm{ks}$, and $30.0 \mathrm{ks}$ for the MOS1, MOS2, and $p n$.

Since we are more interested in studying the relatively high surface brightness regions of A496, where the background subtraction is less critical than for more external low surface brightness regions, we made use of blank-sky fields instead of proceeding with a more detailed modeling of the different background components. The blank-sky fields for EPIC MOS and pn were produced by Leccardi \& Molendi (2008, see Appendix B "The analysis of blank field observations" in their paper) by analyzing a large number of observations for a total exposure time of $\sim 700 \mathrm{ks}$ for MOS and $\sim 500 \mathrm{ks}$ for $p n$. We refined our background analysis by also performing a background rescaling for each observation separately to account for temporal variations of the background. We estimated the background intensity from spectra extracted from an external ring between $10^{\prime}$ and $12^{\prime}$ centered on the emission peak at $(\mathrm{RA}, \mathrm{Dec})=(4: 33: 38 ;-13: 15: 41)$, taking into account only the $10-12 \mathrm{keV}$ band (to avoid possible extended cluster emission residuals in this region). Finally, we rescaled the blank-sky fields background to the local value. The scaling factors are in the range [1.5-1.8]. These values are higher than usual because of the higher threshold adopted for filtering soft protons. This procedure scales both the instrumental and the sky background. However, the sky background is almost negligible in the central regions of the cluster, on which our analysis is focused which means that this procedure does not introduce any significant bias in the measure.

\subsection{X-ray surface brightness image and thermodynamical maps}

Using the cleaned event files for MOS1, MOS2, and $p n$ we built the EPIC flux map: MOS1 + MOS2 + pn (with $p n$ images corrected for out-of-time events). This flux image was computed in the $0.4-2 \mathrm{keV}$ energy band and the two observations were joined to obtain a single mosaicked map. Details on the preparation of the EPIC flux images can be found in G10 and Rossetti et al. (2007). The resulting flux map is shown in Fig. 1. The flux image is in units of $10^{-15} \mathrm{erg} \mathrm{cm}^{-2} \mathrm{~s}^{-1}$ pixel $^{-1}$ (one pixel is $3.25 \times 3.25 \operatorname{arcsec}^{2}$ ).

To study the discontinuities in A496, we also built thermodynamical maps using the WVT + broad band fitting (BBT) method introduced and described in Rossetti et al. (2007), where we used the weighted Voronoi tessellation (WVT) binning algorithm by Diehl \& Statler (2006) in place of the Voronoi binning algorithm by Cappellari \& Copin (2003). We set a minimum value of the signal-to-noise ratio $S / N=10$ for the binning algorithm and used the energy bands $(0.4-0.8 \mathrm{keV}, 0.8-1.2 \mathrm{keV}$, 1.2-2 keV, 2-4 keV, 4-10 keV) for the broad-band fitting procedure. In Fig. 2 we show the maps for the temperature $T$ (panel a), the projected pressure $P$ (panel b), and the projected entropy $K$ (panel c).

As is conventional in X-ray astronomy, we quantified the entropy using the adiabatic constant $K=k T n_{\mathrm{e}}^{-2 / 3}$ ( $T$ and $n_{\mathrm{e}}$ are the gas temperature and density, and $k$ is the Boltzmann constant) following Voit et al. (2005; see also Ponman et al. 2003). The 
specific entropy $s$ is related to $K$ through the relation $s \propto \ln K$. For brevity, we refer to $K$ as entropy throughout the paper. To derive $K$, we should deproject cluster surface brightness and temperature (see e.g. Ghizzardi et al. 2004). However, because of the surface brightness discontinuities, the cluster is asymmetric and the deprojection technique is not particularly useful. For this reason, we used the projected entropy derived as $T / E M^{1 / 3}$ and the projected pressure derived as $T \cdot E M^{1 / 2}$, where $T$ and $E M$ are the temperature and the emission measure (XSPEC normalization per pixel) derived from the spectral fit. Since we used projected quantities, $P$ and $K$ are given in arbitrary units throughout the paper.

\subsection{Spatially resolved spectral analysis}

We performed the spectral analysis of A496 in a series of regions: radial bins along sectors centered on the emission peak (see Sect. 3.3) and other relevant polygonal regions (see Sect. 3.4). In the following we describe how we handled the spectrum of a generic region regardless of its shape.

We extracted the total (i.e., source plus background) spectrum of each region for each observation. The corresponding redistribution matrix files (RMFs) and ancillary response files (ARFs) were generated with the SAS tasks rmfgen and arfgen. The background spectrum was extracted from blank-sky fields as described at the beginning of this section. Before proceeding with the spectral analysis, we co-added, by weighting appropriately, the total and background spectra of the two observations with the FTOOLS matpha, the ARFs with addarf and the RMFs with addrmf. Spectra from all three EPIC instruments were then fitted simultaneously using the spectral band between 0.7 and $8.0 \mathrm{keV}$. All spectral fits were performed with the XSPEC package (version 11.3.2, Arnaud 1996).

We analyzed each cluster spectrum with two different models: a one-temperature thermal model with the plasma in collisional ionization equilibrium (vapec model in XSPEC), referred to as the $1 \mathrm{~T}$ model hereafter, and, a multitemperature model in which the temperature distributions are a Gaussian (GDEM model, as described in Buote et al. 2003 Eq. (3)), where the mean temperature $T_{0}$ is the mean and $\sigma_{T}$ is the standard deviation of the Gaussian.

In the $1 \mathrm{~T}$ model we allowed temperature, normalization, redshift, and elements $\mathrm{Si}, \mathrm{S}$, and Fe to vary freely. $\mathrm{C}, \mathrm{N}$, and $\mathrm{O}$ were fixed at $0.4 ; \mathrm{Mg}$ and $\mathrm{Al}$ were linked to $\mathrm{Ne}$, which was left free to vary; $\mathrm{Ar}$ and $\mathrm{Ca}$ were linked to $\mathrm{S}$ because these elements are less abundant and we did not plan to study them in this work. $\mathrm{Ni}$ was fixed at 1.8 times the value of $\mathrm{Fe}$ following the result found by De Grandi \& Molendi (2009) from a sample of coolcore clusters. In the GDEM model we left as free parameters the mean temperature $T_{0}$ and the $\sigma_{T}$ of the Gaussian temperature distribution and the metallicity. In both models the redshift was always left free, to account for small calibration differences between the two MOS and the $p n$ cameras. We accounted for low-energy absorption by multiplying by the Galactic hydrogen column density, $N_{\mathrm{H}}=3.76 \times 10^{+20} \mathrm{~cm}^{-2}$, determined by HI surveys (Kalberla et al. 2005) through the wabs absorption model in XSPEC. In the following sections we only report results from spectral fits with $N_{\mathrm{H}}$ fixed at the weighted Galactic value. We have also allowed $N_{\mathrm{H}}$ to vary in both models and found no significant differences in the derived temperature and metal abundance values.

As pointed out by Leccardi \& Molendi (2008), when fitting spectra with XSPEC, it is appropriate to allow the metallicities to assume negative values. This procedure is necessary to avoid

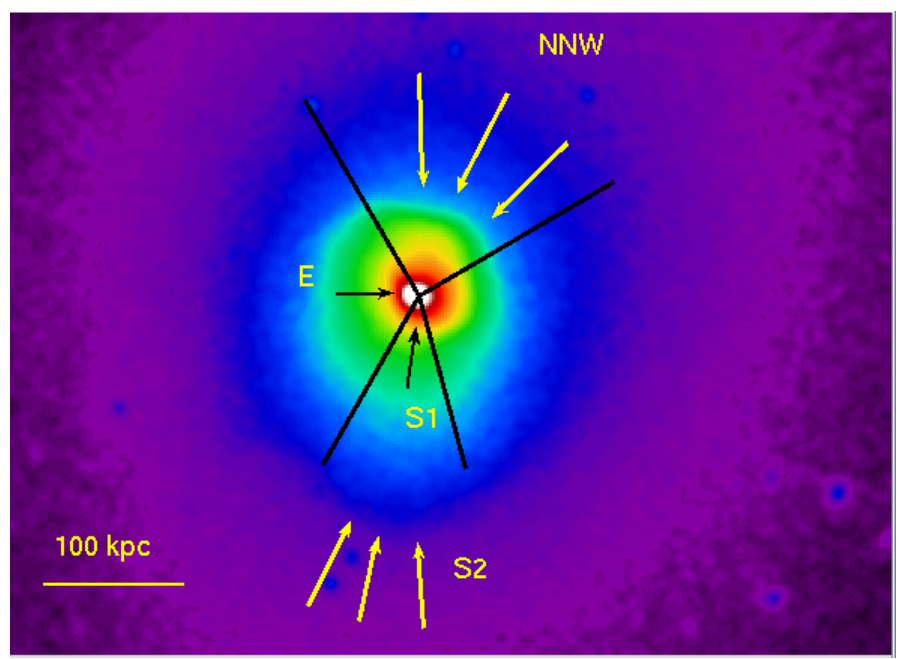

Fig. 1. EPIC surface brightness image in the energy range $0.4-2 \mathrm{keV}$. Black lines define the sectors that host cold fronts (see text for further details). Black arrows mark the position of the inner cold fronts (labeled $\mathrm{E}$ and S1) and yellow arrows the positions of the outermost cold fronts (labeled NNW and S2).

underestimating metal abundances, especially in the case of lowmetallicity statistically poor spectra (for a more detailed discussion of this point see Appendix A in Leccardi \& Molendi 2008). Abundances are measured relative to the solar photospheric values of Anders \& Grevesse (1989), where Fe $=4.68 \times 10^{-5}$, $\mathrm{Si}=3.55 \times 10^{-5}$ and $\mathrm{S}=1.62 \times 10^{-5}$ (by number relative to $\mathrm{H}$ ). We have chosen these values to allow a direct comparison with other works in the literature.

Finally, only for $p n$ we always included a multiplicative component that performs a Gaussian smoothing of the spectral model (gsmooth in XSPEC) to take into account a small miscalibration of the redistribution matrix of this detector as shown first in Molendi \& Gastaldello (2009) and then investigated in detail by De Grandi \& Molendi (2009, see their Sect. 4.1 and Fig. 1).

\section{Results}

\subsection{Four circum-core cold fronts in A496}

A visual inspection of the flux map of Fig. 1 shows a very regular, centrally peaked X-ray surface brightness typical of a coolcore cluster. The surface brightness is fairly elongated along the NW-SE direction. The map clearly reveals several sharp discontinuities. The main discontinuity is located in the NNW direction $\left(30^{\circ}-120^{\circ}\right.$; angles are measured from $\left.\mathrm{W}\right)$ at a distance $\sim 65 \mathrm{kpc}$ $\left(\sim 100^{\prime \prime}\right)$ from the X-ray peak. The front is sharp with the surface brightness dropping by about a factor of 3 in $20 \mathrm{kpc}$ and exhibits a boxy morphology. A surface brightness discontinuity is also observed $\sim 35 \mathrm{kpc}\left(\sim 55^{\prime \prime}\right)$ from the center on the opposite side, in the south direction (labeled S1 in Fig. 1). Both these discontinuities have also been detected and studied by Dupke \& White (2003) and Dupke et al. (2007) using Chandra data. The former authors also detected a third discontinuity located at $\sim 16 \mathrm{kpc}$ (labeled E in Fig. 1) east of the center. This cold front is too close to the center to be resolved by the XMM-Newton instruments.

Another outer discontinuity, marked S2 in Fig. 1, is detected further south, $\left(240^{\circ}-285^{\circ}\right), \sim 160 \mathrm{kpc}\left(\sim 240^{\prime \prime}\right)$ from the center (see also Tanaka et al. 2006). This front could not be detected in Chandra maps because it lies almost at the edge of the ACIS-S3 chip. 


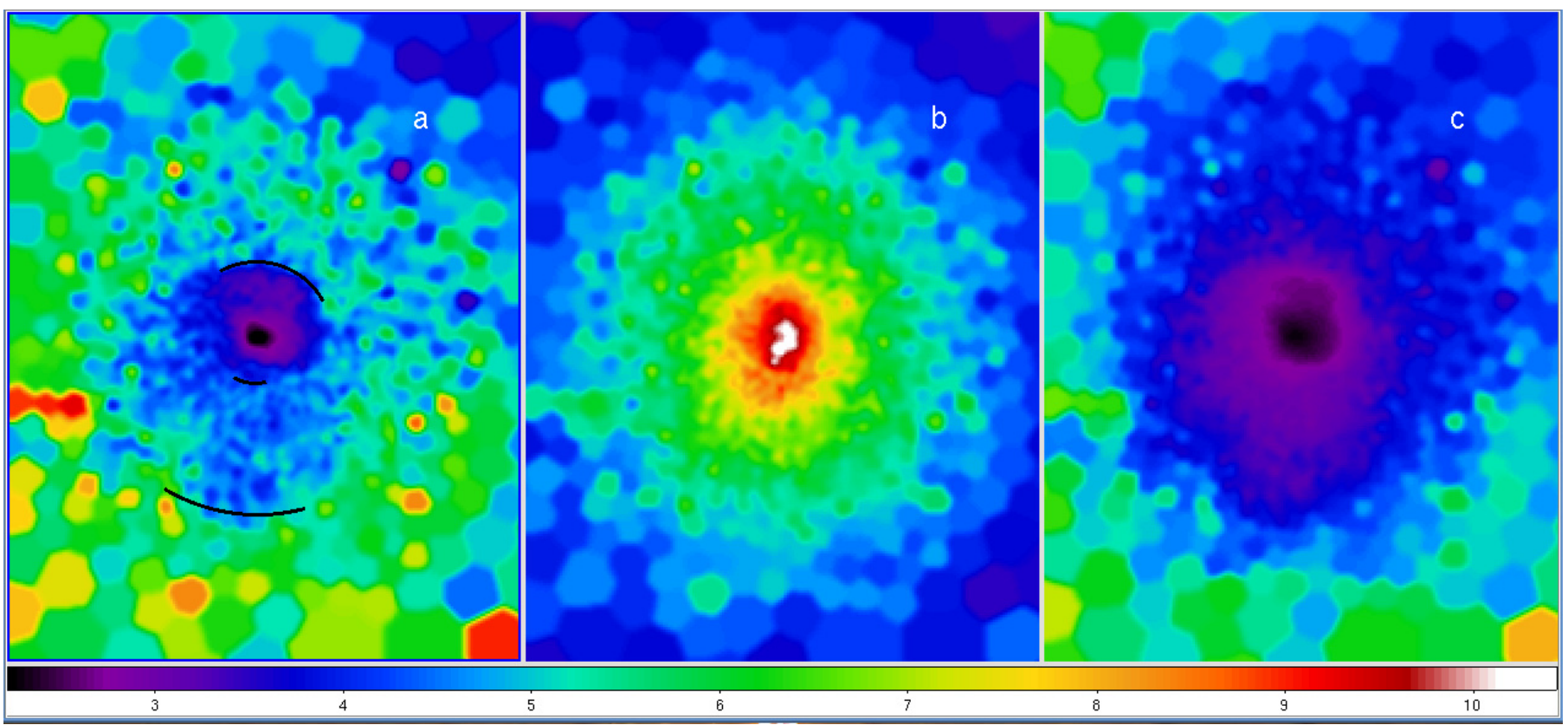

Fig. 2. EPIC temperature a), projected pressure b), and projected entropy c) maps for A496. Maps have been binned using a WVT+BBF algorithm with a $S / N=10$ per bin and then smoothed with a $\sigma=9.75^{\prime \prime}$ Gaussian. Black arcs in panel a) mark the positions of the cold fronts. The color bar indicates the temperature in $\mathrm{keV}$.

In Fig. 2 we show the maps for the temperature (panel a), the projected pressure (panel b) and the projected entropy (panel c). The black arcs in panel (a) mark the position of the discontinuities.

The temperature map confirms that the cluster has a central cool core with a decreasing temperature in the central regions, reaching the value of $\sim 2 \mathrm{keV}$ in the core, starting from an outer value of $\sim 5 \mathrm{keV}$. The map also shows that the cooler gas lies in the higher density side of the surface brightness edges, with the temperature increasing across the discontinuity, as is typical of cold-front features.

The sharpness of the temperature rise across the edges can be better appreciated by looking at the temperature profiles of the sectors that contain the discontinuities. We built the temperature profile of each sector of interest by plotting the temperature of each map bin belonging to the selected sector versus the bin distance from the X-ray peak. In Fig. 3 we plot the temperature profiles for the sectors that host the surface brightness discontinuities. The NNW sector has been split into two subsectors $\left[30^{\circ}-75^{\circ}\right.$ and $\left.75^{\circ}-120^{\circ}\right]$ because of the boxy appearance of the discontinuity. The sector $240^{\circ}-285^{\circ}$ hosts the two southern fronts. The temperature sharply increases across each edge, confirming that all these discontinuities are cold fronts. Notably, the cluster hosts four cold fronts (including the E cold front observed by Dupke \& White 2003; Dupke et al. 2007). Notoriously, the development of multiple cold fronts in a relaxed cluster is likely to be generated by the sloshing of the central cool gas.

Another marker of the occurrence of the sloshing in A496 is the spiral feature in the temperature and entropy maps. Spiral patterns have been observed in the temperature maps of several clusters that host sloshing cold fronts (Clarke et al. 2004; Fabian et al. 2006; Laganá et al. 2010) and are predicted by simulations (Ascasibar \& Markevitch 2006; Roediger \& ZuHone 2012; Roediger et al. 2012). Numerical simulations by Ascasibar \& Markevitch (2006) show that the central cool gas starts sloshing after a minor-merger event. If the cluster is experiencing an off-axis merger, the gas may acquire an angular momentum and the sloshing takes a spiral-like appearance. Panels (a) and (c) of Fig. 2 reveal a hint of such a spiral. The spiraling pattern is remarkably sharp in the entropy residual map (Fig. 4). This map is obtained as $\left(K-K_{\text {ave }}\right) / K_{\text {ave }}$, where $K$ is the entropy map and $K_{\text {ave }}$ is the averaged entropy map obtained by averaging entropy in concentric annuli. Hence by definition, regions that in Fig. 4 are zero correspond to the regions in the cluster whose entropy equals the averaged entropy; darker regions (negative values) are the cluster regions with low entropy levels, and lighter regions (positive values) are the cluster regions with an entropy excess with respect to the average. This method allows highlighting deviations from the averaged values so that asymmetries and patterns can be more easily detected. The entropy residual map shows that the central, cool, low-entropy gas develops in a spiralshaped fashion extending from the center anticlockwise toward north where the main NNW cold front is located; here the spiral turns eastward and finally expands toward the south. Cold fronts are located along the edge of the spiral and the southern outermost cold front (S2) is placed at the tail.

Panel (c) of Fig. 2 shows that the gas entropy steeply drops in the central regions. This is expected in sloshing clusters. Indeed, simulations by Ascasibar \& Markevitch (2006) highlighted that a steep central entropy profile is a necessary condition for triggering the sloshing. On the observational side, by analyzing a large sample of clusters observed with XMM-Newton, G10 found that clusters that host sloshing cold fronts feature entropy drops in their cores, in agreement with theoretical expectations (but see the case of the outermost cold front in A2142; Rossetti et al. 2013).

Unlike the temperature and the entropy maps, the pressure map (panel b in Fig. 2) is regular, nearly symmetric, with a mild elongation along the NW-SE direction. Displacements of the pressure from the averaged value are modest, within $10 \%$ in almost all the regions. This may indicate that the cluster is fairly relaxed and that no remarkable perturbations to the underlying gravitational potential have been induced by any recent major-merger event, as expected in a sloshing scenario, where 

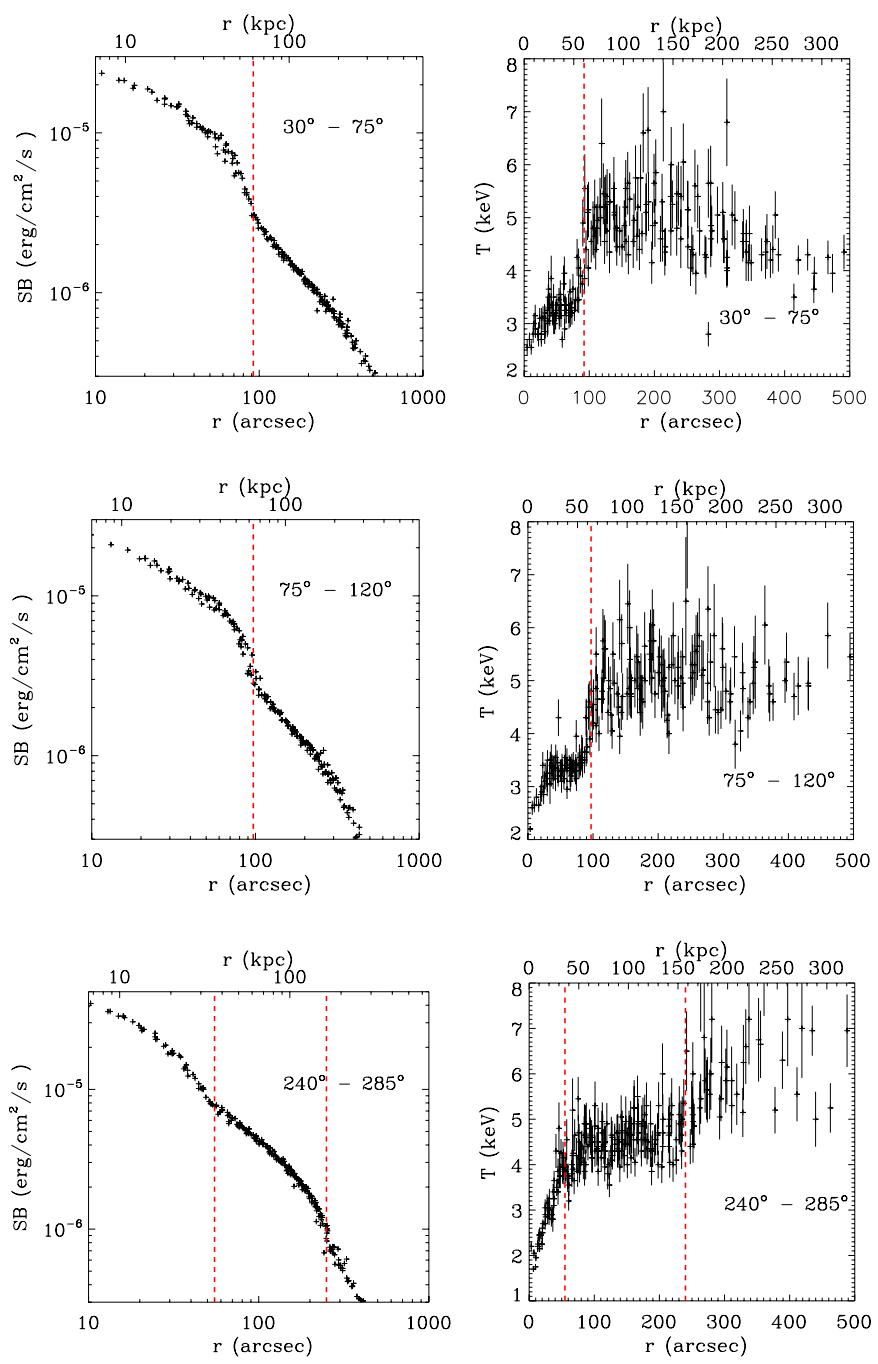

Fig. 3. Surface brightness (left panels) and temperature (right panels) profiles for the sectors that host cold fronts. Profiles are built from the binned maps of Fig. 2. Red dashed lines mark the positions of the cold fronts.

the mechanism is thought to be triggered by minor mergers. The regular configuration of the pressure also confirms that there are no substantial departures from hydrostatic equilibrium and that, if some gas is sloshing, the motion is subsonic. This picture is supported by simulations specifically tailored to A496 (Roediger et al. 2012). The authors reproduce the characteristic spiral pattern and suggest that sloshing has been triggered by a minor-merger subcluster that crossed A496 from the southwest to north-northeast, after its passage southeast of the cluster core.

The analysis of the thermodynamical maps highlights that A496 is a sloshing cluster hosting four cold fronts. So far, this is one of the few clusters where four cold fronts have been detected, together with A2142 (Rossetti et al. 2013; Markevitch, priv. comm.) and Perseus, if its western surface brightness excess detected by Simionescu et al. (2012) is confirmed to be a cold front. Among this handful of clusters, A496 is the only one hosting four cold fronts in a circum-core area $(r<250 \mathrm{kpc})$, while farthest cold fronts of A2142 and Perseus lie at distances $>0.5 \mathrm{Mpc}$. This characteristic makes A496 a unique cluster for investigating sloshing properties.

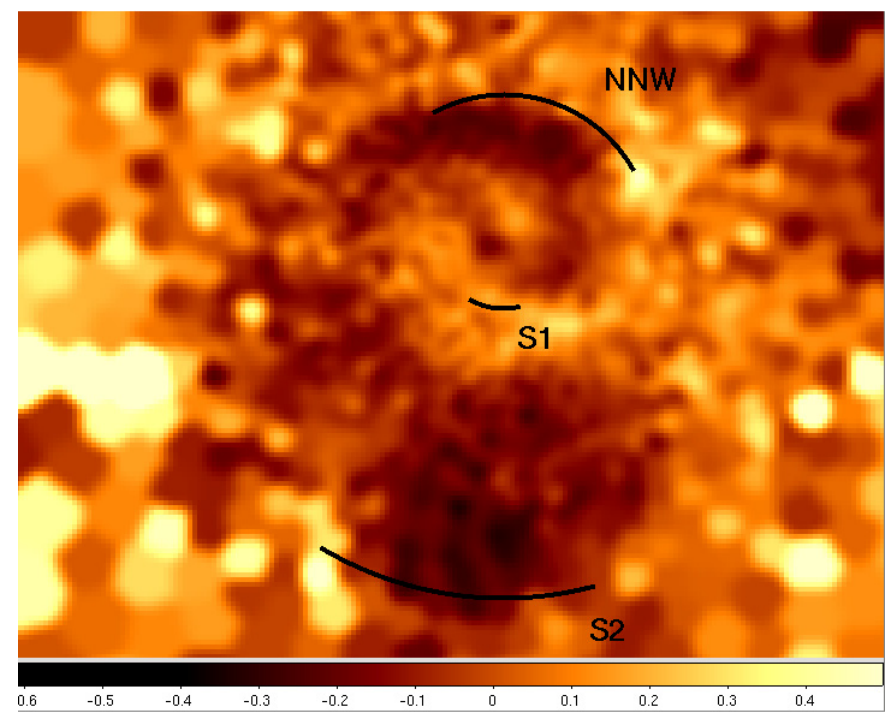

Fig. 4. Entropy residual map. Black arcs mark the detected cold fronts. The residual entropy is zero in regions whose the entropy equals the averaged entropy; darker regions (negative values) have low entropy levels, lighter regions (positive values) have an entropy excess with respect to the average.

\subsection{Characterization of the fronts}

We characterized our cold fronts by means of a broken powerlaw (see G10 for details). The slope and the normalization of each power law are free parameters. We derived the surface brightness by projecting the emissivity along the line of sight, assuming spherical symmetry. We fixed the temperatures inside and outside the fronts using the spectral analysis results (see Sect. 3.3). We fit the observed surface brightness with the projected profiles to derive the parameters of the power laws and find the electron density profile. By using the density and the temperature, it is then straightforward to derive the pressure profile and the pressure jump at the front (see also Appendix B for details). The innermost eastern cold front $\mathrm{E}$ is located at $\sim 16 \mathrm{kpc}$ $\left(\sim 24^{\prime \prime}\right)$ from the center (Dupke et al. 2007), and it is too close to the center to be resolved by the XMM-Newton instruments. The southern $\mathrm{S} 1$ cold front lies at $\sim 35 \mathrm{kpc}\left(\sim 55^{\prime \prime}\right)$ from the center, and although it is clearly detected by XMM, a detailed characterization of the profile is difficult. Therefore, we did not analyze these two fronts and focus on the NNW and S2 cold fronts. We split the NNW cold front into two subsectors because of the boxy morphology of this discontinuity. For the NNW cold front, we measure a pressure jump $p_{\text {in }} / p_{\text {out }}=1.40 \pm 0.03$ in the sector $30^{\circ}-75^{\circ}$, while in the sector $75^{\circ}-120^{\circ}$ we find a slightly lower pressure jump $p_{\text {in }} / p_{\text {out }}=1.33 \pm 0.02$. The front position was set to $92^{\prime \prime}$ and $102^{\prime \prime}$ (corresponding to $\sim 60$ and $65 \mathrm{kpc}$ ) in the two subsectors. Finally, for the cold front S2, we measure a pressure jump $p_{\text {in }} / p_{\text {out }}=1.28 \pm 0.03$ in the sector $240^{\circ}-285^{\circ}$, at the front position $252^{\prime \prime}$ (corresponding to $\sim 165 \mathrm{kpc}$ ). In Fig. 5 we plot the surface brightness profiles of the three sectors with their best fits.

According to Vikhlinin et al. (2001a), the Mach number and the velocity of the cold front can be derived from the pressure jumps, in merging systems. Pressure ratios of about 1.3-1.4, similar to those we have measured, would give Mach numbers $\mathcal{M} \sim 0.6-0.7$. Conversely, velocity estimates cannot be derived directly from pressure jumps for sloshing cold fronts since the cold front moves outward while the gas is spiraling around the center of the potential well; however, we expect that the pressure ratios provide an order-of-magnitude estimate of the real 

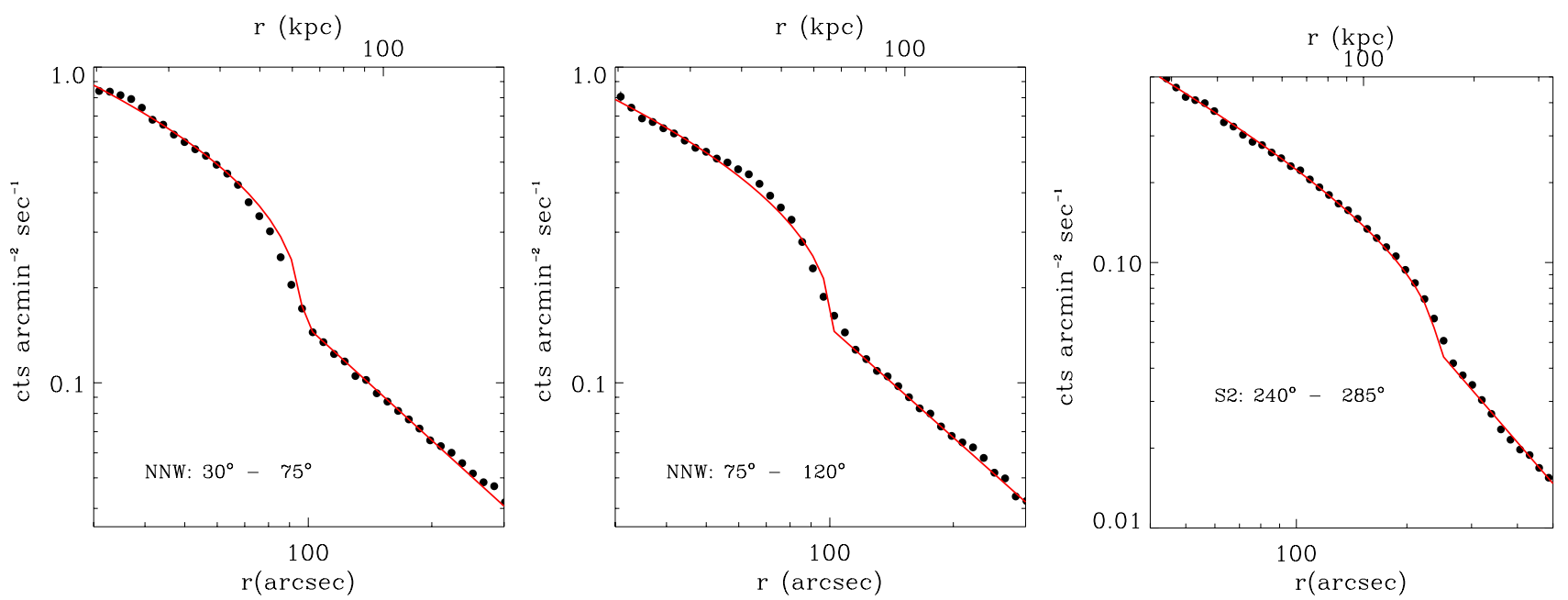

Fig. 5. Surface brightness profiles in the three sectors that host cold fronts: $30^{\circ}-75^{\circ}$ (left panel), $75^{\circ}-120^{\circ}$ (middle panel) and $240^{\circ}-285^{\circ}$ (right panel). The solid red line is the best-fit surface brightness obtained assuming that the electron density follows a broken power-law.

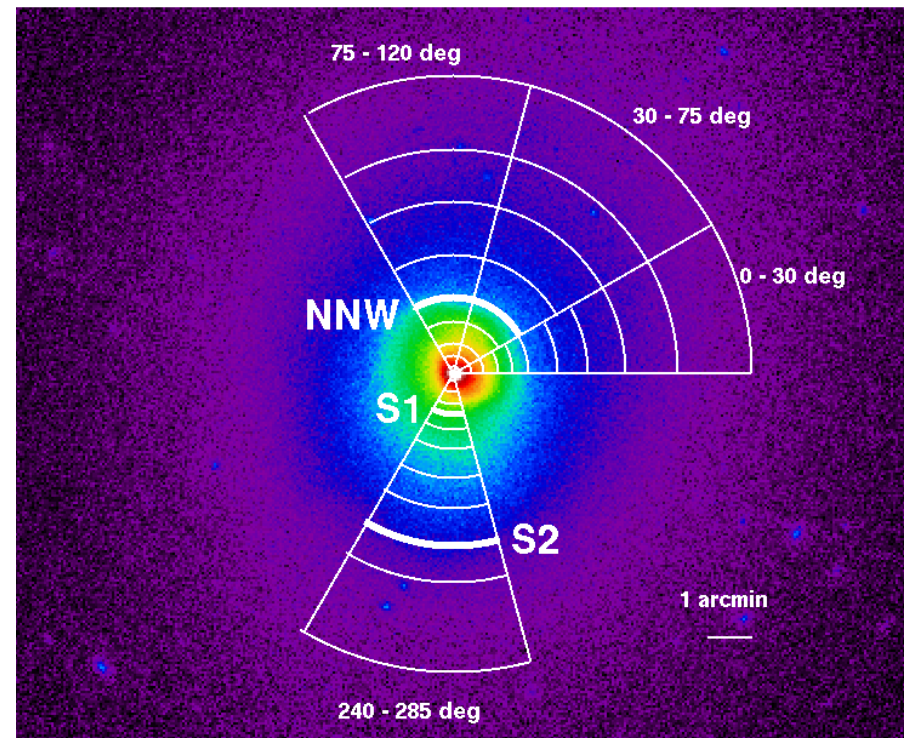

Fig. 6. X-ray surface brightness image (same as in Fig. 1); white overlay shows regions centered on the X-ray peak that was used to derive the profiles presented in Fig. 7. NNW, S1 and S2 label the three cold fronts. The positions of the fronts are marked with a thick arc.

velocities within a factor of 2 . Thus the pressure jumps we detect in A496 imply Mach numbers in the range 0.3-1.0.

\subsection{Radial temperature and metal abundance profiles within sectors}

Starting from the central peak position (RA 04:33:38; Dec -13:15:41) we divided the cluster into eight sectors with bounding angles: $0^{\circ}, 30^{\circ}, 75^{\circ}, 120^{\circ}, 180^{\circ}, 240^{\circ}, 285^{\circ}, 330^{\circ}$, and $360^{\circ}$ (measured from $\mathrm{N}$ to $\mathrm{E}$, with $0^{\circ}$ toward $\mathrm{W}$ ). The radial bins in each sector were chosen to match the expected positions of the cold fronts. In Fig. 6 we show the bins used for the spectral analysis, for the sectors of interest. We extracted spectra for each bin and performed the spectral analysis with the singletemperature (1T) and multitemperature (GDEM) models, as described in Sect. 2.2.
We first searched for evidences of more than one temperature in our spectra. We found that the only regions where the relative temperature and iron abundances of the two models differ, that is where $\left(T_{1 \mathrm{~T}}-T_{\mathrm{GDEM}}\right) / T_{\mathrm{GDEM}}$ and $\left(Z_{\mathrm{Fe}, 1 \mathrm{~T}}-Z_{\mathrm{Fe}, \mathrm{GDEM}}\right) / Z_{\mathrm{Fe}, \mathrm{GDEM}}$, are higher than or equal to $\sim 10 \%$ and $\sim 20 \%$, respectively, correspond to the very central circular bin $\left(0-10^{\prime \prime}\right)$. This is expected because this bin includes the central AGN. The other bins show relative differences in temperature of $\leq 5 \%$ and the iron abundances differences are always consistent within $1 \sigma$.

We concluded that if multiphase gas is present in these bins, it should be extremely limited since the mean temperature found by the GDEM model is indistinguishable (within the systematics of calibration, spectral analysis or projection errors) from the temperature found by the $1 \mathrm{~T}$ model. We refer to Appendix A for a more extended discussion of the possible presence of multiphase ICM in some regions of A496, in the rest of this section we use the results of the $1 \mathrm{~T}$ analysis, except for the central bin, for which we use the temperature and $Z_{\mathrm{Fe}}$ measured with GDEM.

In Fig. 7 we plot the temperature, iron, silicon, and sulfur abundance profiles for the three sectors hosting the NNW $\left(30^{\circ}-75^{\circ}\right.$ and $\left.75^{\circ}-120^{\circ}\right)$ and $\mathrm{S} 2\left(240^{\circ}-285^{\circ}\right)$ cold fronts and, for comparison purposes, a sector $\left(0^{\circ}-30^{\circ}\right)$ where no cold fronts were detected.

All the temperature and $\mathrm{Fe}$ abundance profiles in Fig. 7 show the typical trend of cool-core clusters, namely a temperature increase from $\sim 2.5 \mathrm{keV}$ in the center to $\sim 4.5 \mathrm{keV}$ in the outermost bins, while the iron abundance progressively decreases from its central peak of $\sim 0.9 Z_{\odot}$ to an external value of $\sim 0.4 Z_{\odot}$. In Fig. 7 the NNW cold front (split into sectors $30^{\circ}-75^{\circ}$ and $75^{\circ}-120^{\circ}$ ) is clearly marked by the temperature jump observed at $100^{\prime \prime}$ $(\sim 65 \mathrm{kpc})$. Interestingly, at the same position the Fe abundance drops abruptly: in both sectors at $100^{\prime \prime}$ the Fe abundance shows a decrease significant at more than $5 \sigma$ from the inner to the outer part of the front. We note that there is a similar trend, although less significant, in the $\mathrm{Si}$ and $\mathrm{S}$ profiles as well. The jumps we measure in $\mathrm{Si}$ abundances are significant at least at a $3 \sigma$ level, while jumps in $\mathrm{S}$ abundances are significant at a $\sim 2-3 \sigma$ level.

A closer look at sector $240^{\circ}-285^{\circ}$ in Fig. 7, where the S2 cold front is located, shows that in the region inside the cold front (with radii in the range between $60-150 \mathrm{kpc}$ ), the Fe abundance and possibly the $\mathrm{Si}$ and $\mathrm{S}$ abundances as well remain roughly constant on a plateau of $\sim 0.6 Z_{\odot}$ before the abundances 
sector: $\left[30^{\circ}-75^{\circ}\right]$
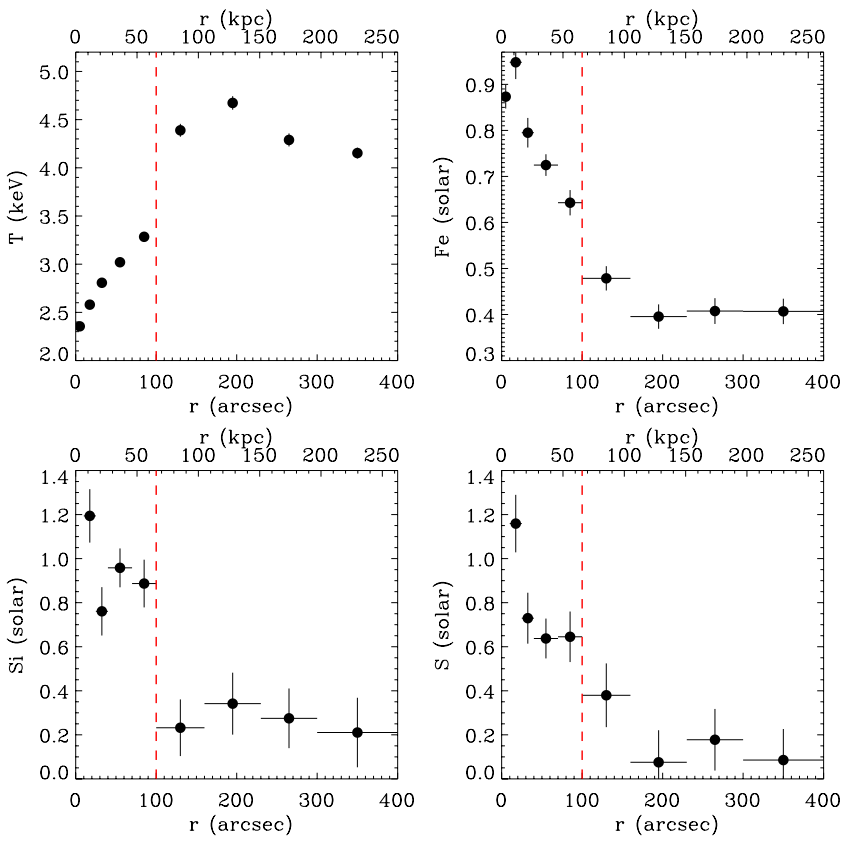

sector: $\left[240^{\circ}-285^{\circ}\right]$
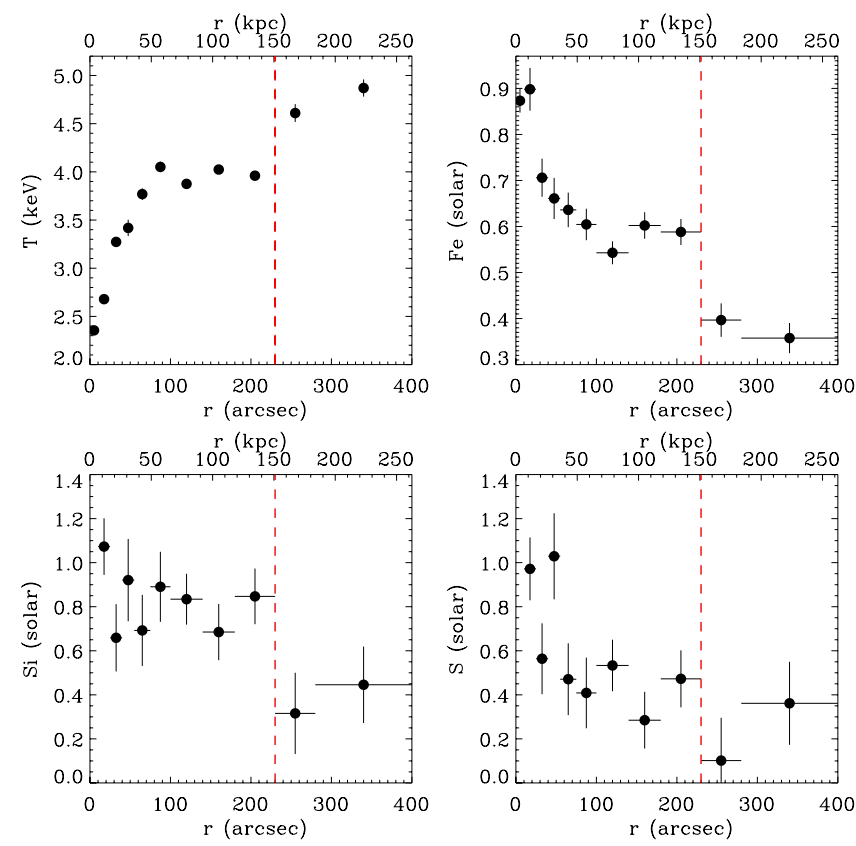

sector: $\left[75^{\circ}-120^{\circ}\right]$
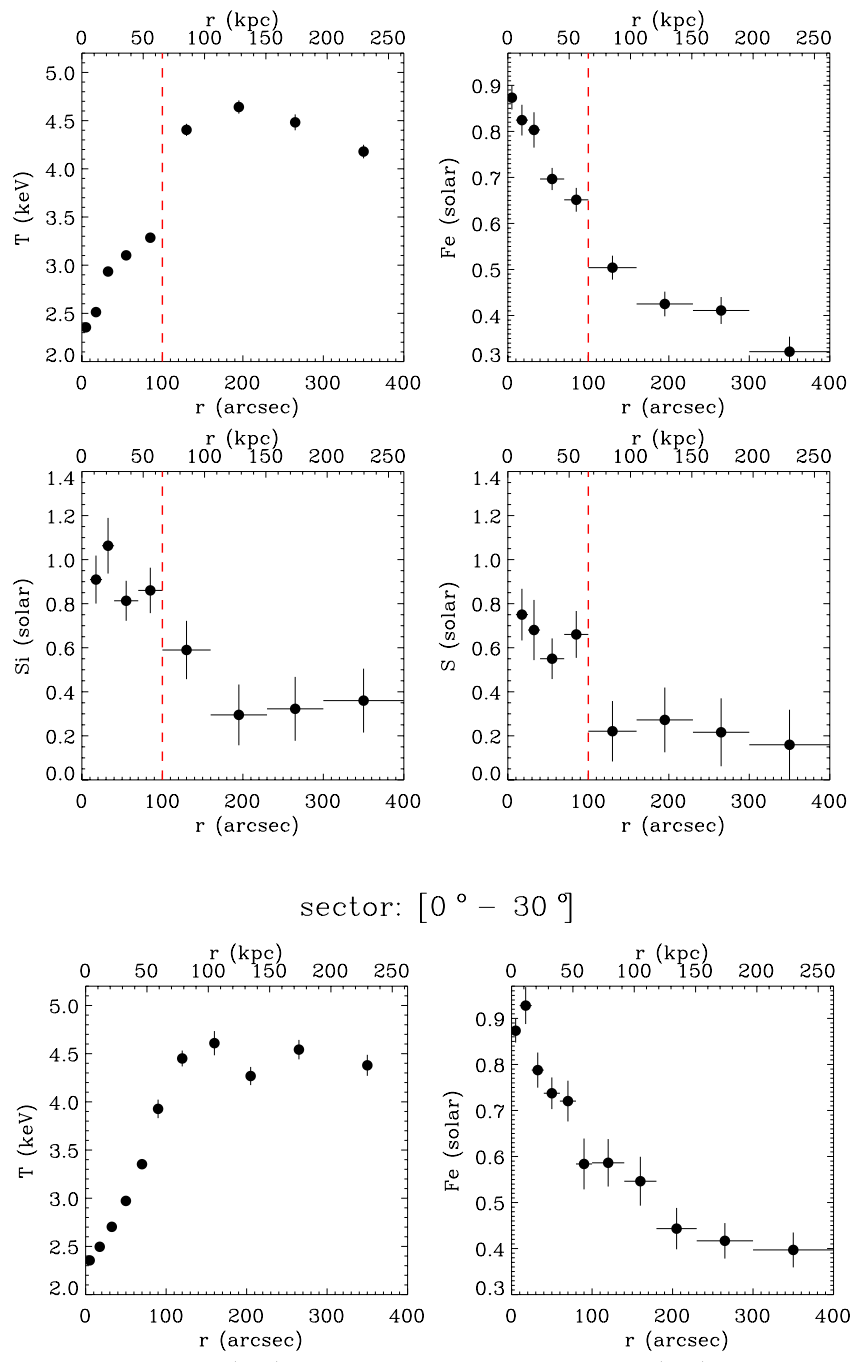

$\left[0^{\circ}-30^{\circ}\right]$
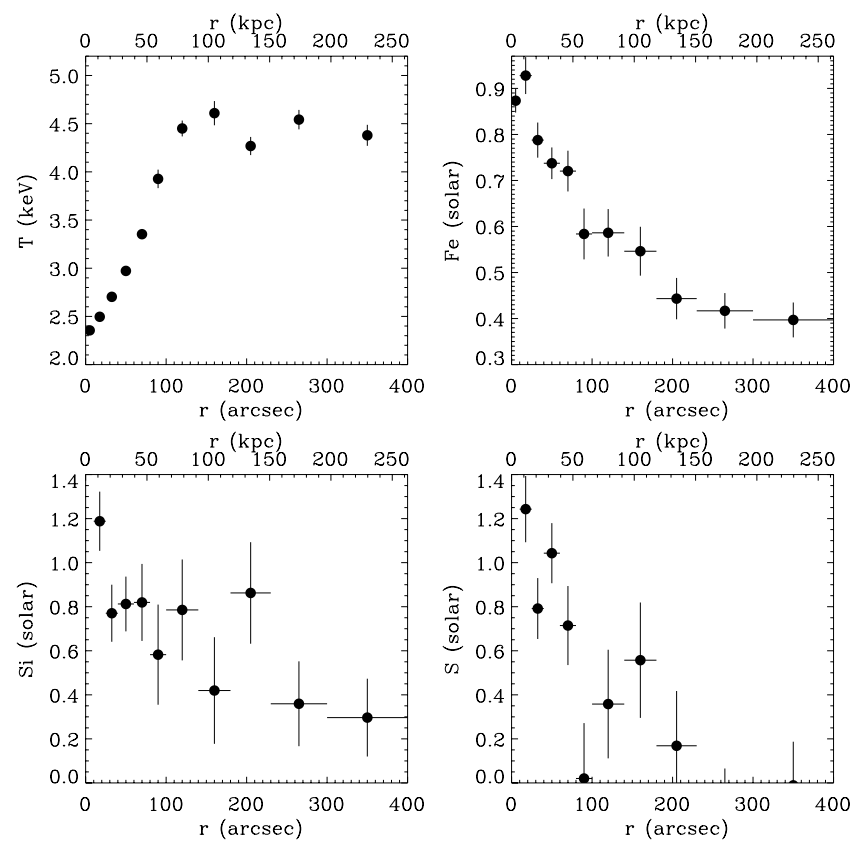

Fig. 7. Temperature, Fe, $\mathrm{Si}$, and $\mathrm{S}$ abundance profiles for the four sectors $30^{\circ}-70^{\circ}$ (top left), $70^{\circ}-120^{\circ}$ (top right), $240^{\circ}-285^{\circ}$ (bottom left) and $0^{\circ}-30^{\circ}$ (bottom right). Red dashed lines mark the cold-front position.

drop at the position of the cold front. This area corresponds to the spiral tail (see Sect. 3.1).

The metal profile in $0^{\circ}-30^{\circ}$ (Fig. 7) is roughly regular, with the metallicity gradually decreasing from the central peak to the outskirt value. The iron abundance profile seems to have some rapid decrease at radius $r \sim 65 \mathrm{kpc}$. The iron abundance varies from $Z_{\mathrm{Fe}}=0.72_{-0.04}^{+0.05} Z_{\odot}$ to $Z_{\mathrm{Fe}}=0.58_{-0.05}^{+0.06} Z_{\odot}$. This decrease cannot be classified as a discontinuity, but we note that its position matches the western edge of the spiral (see Fig. 4). In spite of the temperature and entropy spiral edge, in this sector we do not detect a surface brightness front at the resolution level of XMM-Newton. No surface brightness front has been detected in that direction even using Chandra high-resolution data (Dupke et al. 2007). The discontinuities might be washed out by Kelvin-Helmoltz (KH) instabilities (see Sect. 4.3 and Fig. 13) that smear the front and reduce the jump amplitudes.

\subsection{Metals and spiral pattern}

Both the metal discontinuity across the fronts (which match the spiral edges) and the metal excess in the wide region of the spiral tail point to a connection between the spiral and the areas with high metallicity. We performed a spectral analysis to investigate this possible correlation. 


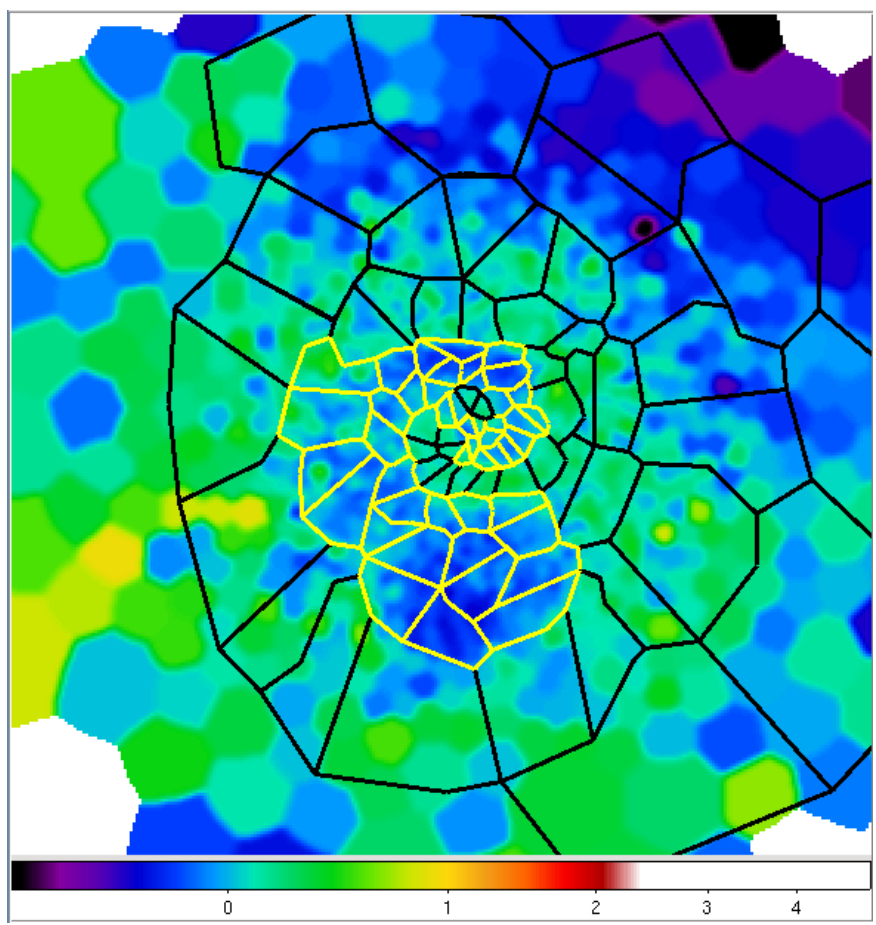

Fig. 8. Entropy residual map with polygons used for the spectral analysis. Yellow labels the IN and black the OUT polygons. The entropy map is the same as in Fig. 4 with the color scale adjusted so that blue regions have negative residual entropy and green regions positive residual entropy values.

We used the entropy residual map (Fig. 4) to distinguish between polygonal regions on the spiral pattern and polygonal regions outside the spiral. We built polygons for spectral analysis by grouping the polygons obtained from the WVT binning. The grouping follows the spiral pattern and maintains a roughly constant $\mathrm{S} / \mathrm{N}$. The extracted spectra typically have 6000-10000 counts in each MOS and 12000-20000 counts in pn. Each spectral polygon is classified as a polygon inside the spiral (IN) or outside the spiral (OUT). Spectral polygons are shown in Fig. 8.

As outlined in the previous section, the ICM spectra are well described by a 1T model except for the central bin (see also Appendix A for the details). Hence, spectra extracted from the polygons were fit with a single temperature vapec model. Spectrally measured temperatures agree well with those obtained using the WVT+BBT algorithm. Figure 9 shows the metal abundance map. The spiral pattern is visible. High-metallicity polygons (white and red in the map color scale) follow the lowentropy spiral configuration; this shows that the metal rich gas does lie on the spiral.

The correlation between low entropy and metal excess may be understood under the assumption that prior to the onset of the sloshing the metal-rich low-entropy gas was located at the bottom of the potential well, that is at the center of the system. After the gas was set into motion, presumably by a perturbing subsystem, the low-entropy metal-rich gas moved outward generating cold fronts and eventually forming the spiral structure. The difference in entropy and metallicity between the gas within and outside the spiral indicates that the processes that mix the lower entropy higher metallicity gas with the ambient medium are relatively ineffective.

The metallicity map (Fig. 9) also highlights a high metallicity region in the SE part of the cluster (orange tones in the map color scale). This point is discussed below (see Sect. 4.7).

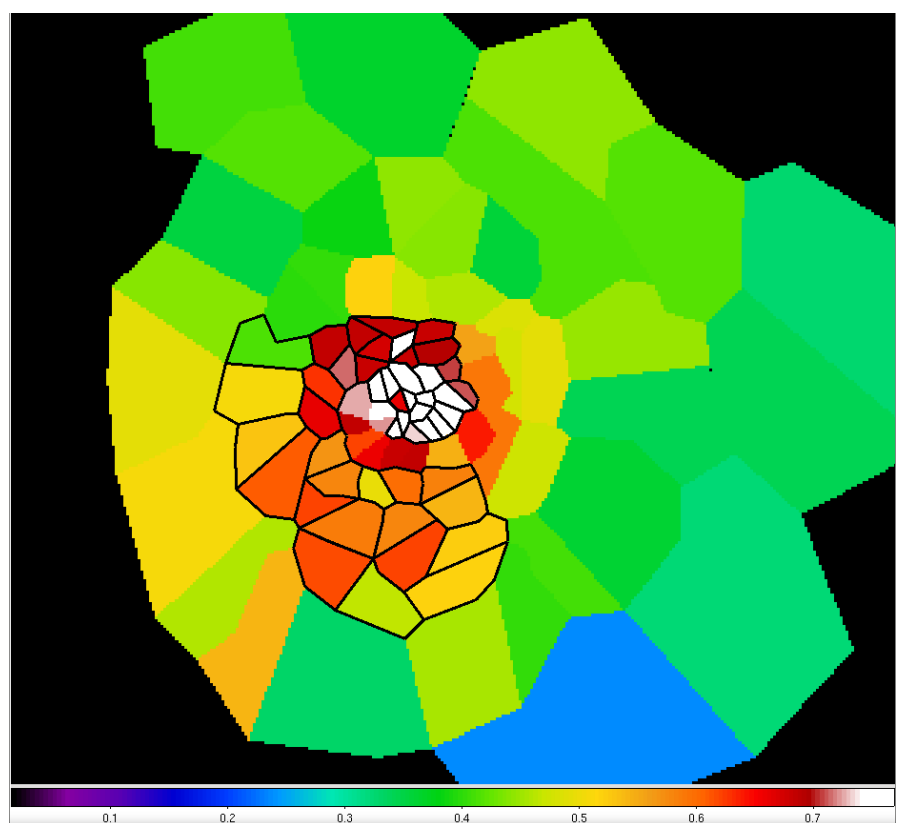

Fig. 9. Iron abundance map obtained from spectral analysis. The color bar indicates the metal abundance in solar units. IN polygons are overlaid.

For a better visualization of the differences between the properties of the gas within and outside the spiral, we plot the thermodynamical quantities of each polygon as functions of its distance from the X-ray peak. Temperature and entropy profiles are reported in Figs. 10 and 11. We recall that we used projected quantities as explained in detail in Sect. 2.1. For a given radius, the entropy and the temperature are generally lower in the IN regions than in the OUT regions. Figure 12 shows the iron abundance profiles. The metal abundance of OUT polygons follows the typical behavior of a cool-core cluster with the metallicity peak in the center and the decreasing trend toward the outer regions. The metallicity of the IN polygons has a similar trend, but offset high with respect to the one measured for OUT polygons. The gas on the spiral tail (red points in the radial range 120"-240" corresponding to $\sim 80-160 \mathrm{kpc}$ ) has a metal abundance $Z_{\mathrm{Fe}} \sim 0.6 Z_{\odot}$, which is lower than the peak value $Z_{\mathrm{Fe}} \sim 0.8-0.9 Z_{\odot}$ and similar to the one observed some $\sim 80^{\prime \prime}-100^{\prime \prime}(\sim 50-65 \mathrm{kpc})$ from the center in other azimuthal directions. Under the assumption that the metal mixing is inefficient, this would imply that the gas on the spiral tail does not come from the center of the cluster, but from an intermediate position, $\sim 50-65 \mathrm{kpc}$ from the center.

\section{Discussion}

\subsection{Iron abundance and sloshing}

The spiral pattern of multiple cold fronts is a clear signature of the onset of the sloshing mechanism in the central regions of the clusters. Our results show that the iron abundance sharply drops across the detected cold fronts (see Fig. 7). A less significant drop is also seen in silicon and sulfur. This metal discontinuity across the front can be promptly explained within the sloshing scenario: when the minor merger triggers the sloshing mechanism, the low-entropy central gas is displaced from the center and pushed out generating the contact discontinuity, that is the front. This dense and cool gas comes into contact with the less dense, hotter ambient gas further enhancing the contrast in density (and surface brightness) and temperature. Assuming that 


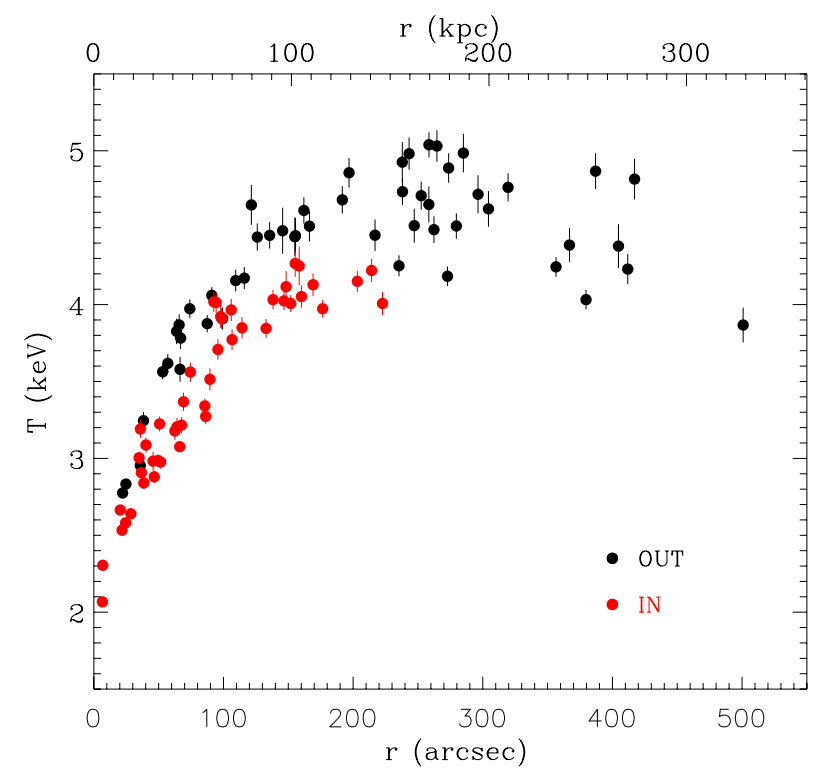

Fig. 10. Temperature profile derived from spectral analysis in the polygonal regions. Red marks the IN, black the OUT polygons.

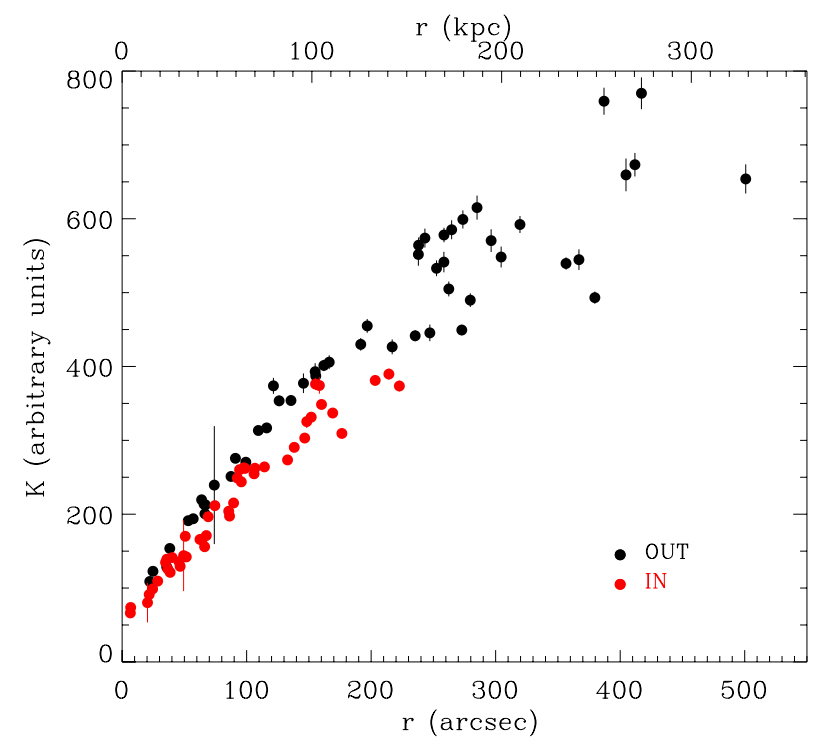

Fig. 11. Entropy profile derived from spectral analysis in the polygonal regions. Red marks the IN, black the OUT polygons.

prior to the onset of the sloshing process the core of A496 was characterized by a metal abundance excess, a metal discontinuity will be created along with the surface brightness and the temperature jumps.

The metallicity drop across the discontinuity is observed in several sloshing clusters (e.g., Perseus: Fabian et al. 2011; Centaurus: Sanders \& Fabian 2006; A2204: Sanders et al. 2005, 2009). In addition to the metal abundance discontinuity across the front, A496 has a notable characteristic for the metal distribution: high-metallicity regions are arranged following the same spiral feature as traced by the low-entropy gas, as apparent in Fig. 9. This is highlighted in Fig. 12, where regions located on the spiral have a higher metal abundance than the ambient gas at the same distance from the center. The most straightforward interpretation is that the sloshing process allows the low-entropy metal-rich gas located at the center to be moved outward. The spiral shape is likely caused by the angular momentum in the

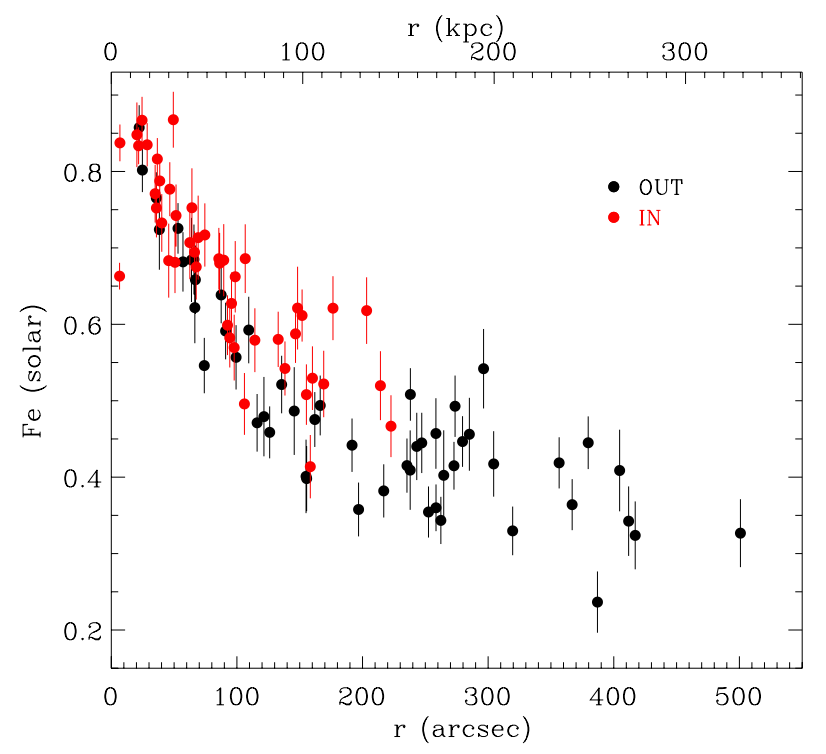

Fig. 12. Fe abundance profile derived from spectral analysis in the polygonal regions. Red marks the IN, black the OUT polygons.

ICM, transferred from the subcluster to the core gas during the off-axis merger (Ascasibar \& Markevitch 2006; ZuHone et al. 2011).

Remarkably, the discrepancy between the metallicity of the gas on the spiral and of the environment gas at the same radius increases with distance: the gas on the tail of the spiral, $\sim 100-150 \mathrm{kpc}$ from the center has a metallicity $\sim 0.6 Z_{\odot}$, which is significantly higher than the averaged metallicity of the hotter surrounding medium $\left(\sim 0.4 Z_{\odot}\right)$. This means that mixing processes are not very efficient. If mixing were efficient, the cool gas would gradually reduce its metal abundance while sloshing and would progressively tend to homogenize with the surrounding. In this scenario, the gas currently residing in the spiral tail would be initially located in the very central regions $(\$ 50 \mathrm{kpc})$ where the environment metallicity is $\gtrsim 0.6 Z_{\odot}$; the higher the mixing efficiency, the higher the starting metal content. Alhough the mechanism would involve all the gas of the spiral, the gas of the tail would cover the largest distance and would undergo the mixing process for a longer time. Hence in presence of efficient mixing processes, the gas of the tail is expected to experience a higher level of mixing and reach a higher degree of homogenization with the ambient gas. This is in conflict with the results shown in Fig. 12, where the largest discrepancy between the IN and OUT gas is observed in the tail.

Assuming an absence of mixing, we can therefore infer that the gas located on the spiral tail did not originate from the the center, but probably comes from an intermediate region, 50-60 kpc. This agrees with simulations by Ascasibar \& Markevitch (2006, see Fig. 8 in their paper), who showed that the relative radial displacement $r / r 0$, (i.e., the ratio between the final distance and starting distance from the center) of the sloshing gas is about $1.5-2$.

\subsection{Velocity and age of the cold fronts}

In sloshing systems, the cool gas moves subsonically in the ambient ICM, with relatively low Mach number values. Although the velocity of the front cannot be directly obtained from pressure ratios in these systems (see Sect. 3.2 for details), the pressure jump can provide a rough estimate (within a factor of 2 , 


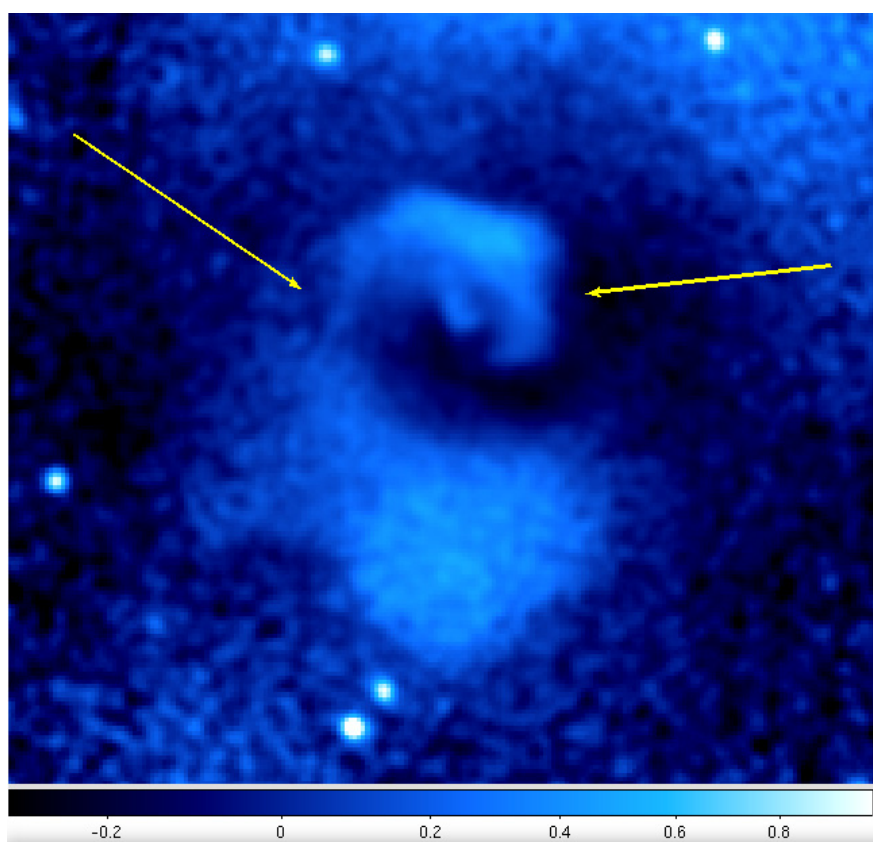

Fig. 13. Surface brightness residual map. Arrows mark the regions where the spiral edges are smeared out, possibly by Kelvin-Helmholtz instabilities.

Vikhlinin \& Markevitch 2002) of the real velocity and, consequently, of the age of the cold front. Assuming a Mach value $\simeq 0.5$, which corresponds to a cold-front velocity of $\sim 500 \mathrm{~km} \mathrm{~s}^{-1}$, in the hypothesis of an averaged ICM temperature $\sim 4 \mathrm{keV}$, the front would cover a distance of $\sim 150 \mathrm{kpc}$ from the center in about $\sim 0.3$ Gyr.

Simulations by Ascasibar \& Markevitch (2006, see their Figs. 3 and 7) show that the time necessary for the central gas to turn back and wrap around to form the central ring of the spiral and for the outermost gas to form the spiral tail is typically 0.6-0.7 Gyr after the subcluster pericentric passage.

Consistently, Roediger et al. (2012) developed highresolution hydrodynamical simulations tailored to reproduce the history of A496 to study the evolution of the cold fronts in this cluster. They found that the sloshing for A496 was probably induced by a perturbing subcluster that crossed from the SW to the NNE, 0.6-0.8 Gyr ago.

\subsection{Kelvin-Helmoltz instabilities}

The spiral pattern drawn by the cool, low-entropy gas is also clearly visible in the residual surface brightness map (see Fig. 13). The figure reveals a noteworthy feature: the spiral portion east of the center that connects the main NNW cold front and the tail appears to be significantly thinner than elsewhere. The same occurs on the spiral section west of the center. The edges of the spiral there are smeared out so that the spiraling feature is fuzzy. A similar configuration can be seen in the maps of Ascasibar \& Markevitch (2006, see their Fig. 7, left bottom panel). This smearing of the front could be explained by KelvinHelmoltz (KH) instabilities. Although cold fronts are stable phenomena, shear flows can be strong enough to trigger KH instabilities. These instabilities tend to create kinks and distortions in the smooth arc-like profile of the fronts and the spiral. The A496 spiral is characterized by features that are similar to those produced by $\mathrm{KH}$ instabilities in simulations (see Roediger et al. 2013; ZuHone et al. 2013b). The elongated shape of A496 probably enhances these features as the elongation stretches the spiral.

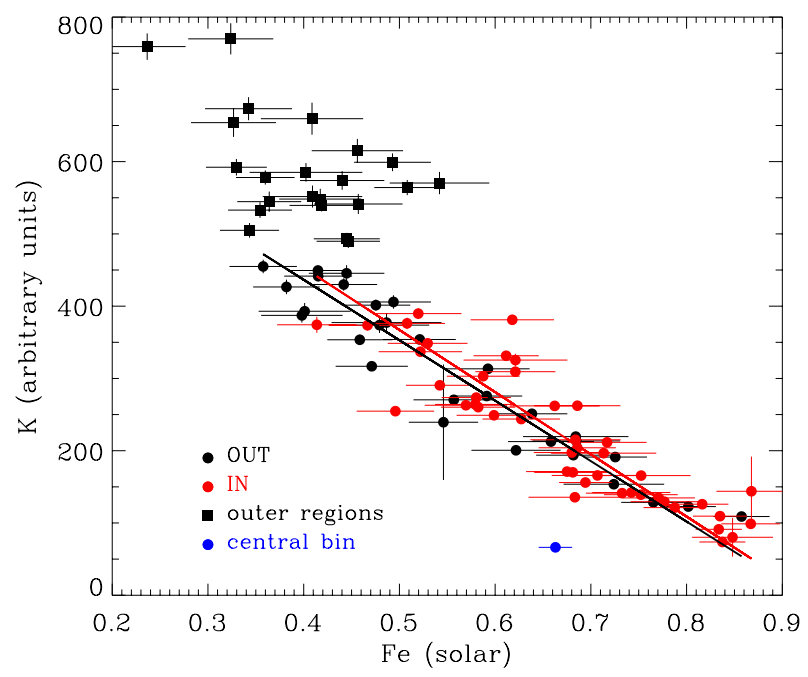

Fig. 14. Entropy - Fe abundance correlation. Red marks the IN polygons, black marks the OUT polygons. The red and black lines are the best fits for IN and OUT points, respectively. The best fits have been derived using the BCES estimator after excluding the outermost regions (black squares) and the spectrally complex central bin (blue point).

\subsection{Entropy and metal abundance correlation}

Fig. 14 shows the well-known correlation (De Grandi et al. 2004; Leccardi et al. 2010) between the metallicity and the gas entropy (K-Fe hereafter). We excluded from our analysis the outlying blue point that corresponds to the spectrally complex central bin (see Appendix A for details). Outer regions deviate from the correlation: at these distances the entropy continues to increase while the metallicity reaches a constant value $\left(\sim 0.3-0.4 Z_{\odot}\right)$. The remarkable result is that the regions IN the spiral appear to follow the same relation as the regions OUT of the spiral. We have quantified this qualitative result with the BCESestimator fitting routine (which allows best-fit determination for measurements with errors in both variables; Akritas \& Bershady 1996). Fitting with $K=K_{0}+r \mathrm{Fe}$ we find $K_{0}=798 \pm 45$ and $r=-861 \pm 65$ for IN regions and $K_{0}=771 \pm 29$ and $r=-836 \pm 51$ for OUT regions. We point out that outer regions where the metal abundance levels off were excluded from the fit; best fits are also shown in Fig. 14.

In light of the striking similarity of the K-Fe relation for IN and OUT regions, we verified whether it may be spurious in nature. More specifically, we checked that the observed IN K-Fe relation is not caused by the neglection of multiphase structure in our spectral analysis. We refer to Appendix $\mathrm{C}$ for a detailed description of this verification. The fact that the sloshing mechanism does not destroy the correlation between metal abundance and entropy has far-reaching consequences which that are discussed in detail in the next section.

\subsection{Thermodynamical processes at work in the ICM}

During the sloshing process, mechanisms that might erase the density and temperature differences between the different phases of the plasma that come into close contact must be suppressed.

The main processes able to erase temperature differences on short timescales are conduction and convection. The last process operates on the gas by physically mixing the two different components, while thermal conduction brings the gas to the thermal equilibrium through heat exchange without mixing the gas particles. Thermal conduction is the most efficient mechanism 
to erase temperature differences. In the Spitzer (Spitzer 1962) regime, thermal conduction operates on a timescale of

$t_{\mathrm{S}} \sim k \ell^{2} n_{\mathrm{e}} / \kappa_{\mathrm{S}} \sim 1.3 \times 10^{-2} n_{\mathrm{e}, 2} \ell_{100}^{2}\left(T_{10}\right)^{-5 / 2} \mathrm{Gyr}$

(Markevitch et al. 2003a; Rasmussen et al. 2006), where $k$ is the Boltzmann constant, $\kappa_{\mathrm{S}}$ is the Spitzer heat conductivity, $T_{10}$ is the temperature in units of $10 \mathrm{keV}, \ell_{100}$ the region width $\ell$ in units of $100 \mathrm{kpc}, n_{\mathrm{e}, 2}$ the electron density $n_{\mathrm{e}}$ in units of $2 \times 10^{-3} \mathrm{~cm}^{-3}$. In our case, $\ell$ can vary from few $\mathrm{kpc}$ (the typical width of a cold front if we wish to estimate the timescale to erase discontinuities) to some tens of $\mathrm{kpc}$ if we wish to bring the whole spiral in thermal equilibrium. The spiral reaches the maximum width in the tail, with a radius of $\sim 60 \mathrm{kpc}$. The electron density is about $n_{\mathrm{e}} \sim 3 \times 10^{-3} \mathrm{~cm}^{-3}$ at the NNW cold front, while the southern outermost cold front at $150 \mathrm{kpc}$ has a density of $n_{\mathrm{e}} \sim 1.5 \times 10^{-3} \mathrm{~cm}^{-3}$. To derive a rough estimate of $t_{\mathrm{S}}$ for the IN gas, we assumed $n_{\mathrm{e}}=2 \times 10^{-3} \mathrm{~cm}^{-3}$, and $T=4.5 \mathrm{keV}$ and $\ell_{100}$ spanning the range [0.06-0.6]. In this hypothesis, the cold fronts would be erased within $t_{\mathrm{S}}=3.3 \times 10^{-4} \mathrm{Gyr}$, (assuming $\left.\ell_{100}=0.06\right)$ and the gas in the spiral would reach thermal equilibrium with the environment within $t_{\mathrm{S}}=3.3 \times 10^{-2} \mathrm{Gyr}$ $\left(\ell_{100}=0.6\right)$. As pointed out in Sect. 4.2 , the lifetime of the cold fronts and the spiral structure in A496 is about 0.6 Gyr, three orders of magnitude longer than the time required to erase the cold-front features and one order of magnitude longer than the time necessary to reach thermal equilibrium and erase the spiral structure: the spiral would be quickly destroyed if conduction were not suppressed along all the spiral edge. While suppression of conduction at cold fronts has been reported by several authors (e.g., Ettori \& Fabian 2000; Vikhlinin et al. 2001a; Gaspari \& Churazov 2013; Gaspari et al. 2014), this is, to the best of our knowledge, the first time that suppression is reported between gas within and outside of the spiral. Magnetic fields are probably responsible for this suppression. Magnetic fields with strengths of about $\mu \mathrm{G}$ are commonly observed in galaxy clusters (Carilli \& Taylor 2002; Ferrari et al. 2008, for recent reviews). Radio mini-halos are also observed in cool cores of sloshing clusters (Govoni et al. 2009; Giacintucci et al. 2011, 2014), suggesting a possible association between the radio emission and sloshing cold fronts (Mazzotta \& Giacintucci 2008; ZuHone et al. 2011, 2013a). Simulations (Asai et al. 2005, 2007; Lyutikov 2006; Dursi \& Pfrommer 2008) show that sloshing can amplify the magnetic field strength up to an order of magnitude and that magnetic fields drape around the front surfaces inhibiting the conduction and the heat exchange across the front.

Various mechanisms can, potentially, operate on the entropy and metal abundance of the sloshing gas. Conduction, if it were to occur at the Spitzer level, would rapidly transfer heat from the hotter environment to the cooler sloshing gas. Our calculations show that the typical timescale for heat transfer over scales of few tens of kpc is significantly shorter than the dynamical timescales associated with the sloshing process. In other words, the motions are sufficiently slow that, as the sloshing gas moves back and forth, it should suffer significant heating from the ambient ICM. Effective heating would therefore move gas in the spiral away from the K-Fe correlation defined by gas outside the spiral. Unless, of course, heating were accompanied by dilution of the IN gas with OUT gas at just the correct rate to maintain the IN points on the K-Fe correlation defined by OUT points. Assuming that conduction is significantly suppressed, mixing of IN and OUT gas could both heat the IN gas and dilute its metal content. However, it would have to operate on spatial scales we cannot resolve (smaller than a few kpc) and, most importantly, the mixing would have to occur at just the correct rate because the IN gas must move along the K-Fe correlation defined by the OUT gas. Moreover, 1) the same processes that inhibit heat transfer by more than one order of magnitude would likely suppress mixing; and 2) as already pointed out in Sect. 4.1, continuous mixing of the IN with OUT gas should lead to a smaller difference between IN and OUT metallicity at larger radii, where the IN gas would have had more time to mix with the OUT gas. In other words, we would need a complex mechanism that is fine-tuned to keep the points on the K-Fe correlation. It must also be noted that this mechanism should at the same time preserve the discontinuities we observe at the cold fronts. The alternative, namely that regions on the spiral lie along the relation defined by regions outside the spiral because both conduction and mixing mechanisms are heavily suppressed, seems to provide a much simpler and natural explanation.

Some MHD simulations (e.g., ZuHone et al. 2013b) show that some anisotropic conduction can be present in the ICM, allowing heat exchange and entropy increase for the cool gas, preserving the characteristic sharp (although reduced) jumps. However, if such a mechanism is indeed operating, it must be rather ineffective, otherwise it would remove the IN regions from the K-Fe relation defined by OUT regions.

\subsection{Sloshing and heating}

Sloshing may contribute to the heating of the cooling core. A possible way is through turbulence: sloshing gas motions may induce turbulence and turbulent energy may be dissipated into heat. However, simulations show that turbulent energy in sloshing cores is a negligible fraction of the thermal energy (Vazza et al. 2012; ZuHone et al. 2013a) and that turbulent diffusion is low (Vazza et al. 2012). While this mechanism could be responsible for the radio mini-halos in sloshing cores they most likely contribute little to the core heating. An alternative way to quench the cooling in a sloshing scenario is through mechanical energy transferred to the ambient ICM via $p \mathrm{~d} V$ work. During the sloshing, the central cool gas is progressively lifted and comes into contact with the hotter environment, which is less dense and has a lower pressure. Gradually, the cooler and denser gas will expand adiabatically to achieve pressure equilibrium with the surrounds. While expanding, the gas does $p \mathrm{~d} V$ work on the surrounding atmosphere. We used our observation to test whether the expanding gas can provide sufficient $p \mathrm{~d} V$ work to offset radiative cooling. We estimated the mechanical power exerted by the sloshing gas and transferred on the environment by following the evolution of the gas currently residing in the tail of the spiral. Under the assumption that the sloshing process is close to adiabatic, this gas must have originally resided some $60 \mathrm{kpc}$ from the center. By deprojecting and assuming entropy conservation, we derived that the work done by the gas rising from $60 \mathrm{kpc}$ to $150 \mathrm{kpc}$ is about $W=\int p \mathrm{~d} V=8.08 \times 10^{58} \mathrm{erg}$. The estimated timescale for the mechanism is $t \sim 0.7 \mathrm{Gyr}$, providing a power $W / t \sim 3.66 \times 10^{42} \mathrm{erg} / \mathrm{s}$. Using the deprojection algorithm (Ghizzardi et al. 2004; Ettori et al. 2002; Ettori 2002), we also evaluated the bolometric luminosity within the cooling radius $(\sim 75 \mathrm{kpc})$ and obtained $L_{\text {cool }}=5.16 \times 10^{43} \mathrm{erg} / \mathrm{s}$, which is more than one order of magnitude larger than the mechanical power supplied by the expanding gas. The cooling cannot be quenched by the sloshing mechanism. In addition, if the sloshing and the ambient gas do not mix significantly, as our results suggest, the cool gas will eventually sink back to the bottom of the potential well. During this reverse process, the low-entropy gas will be compressed by the ambient medium and, in the adiabatic limit, 


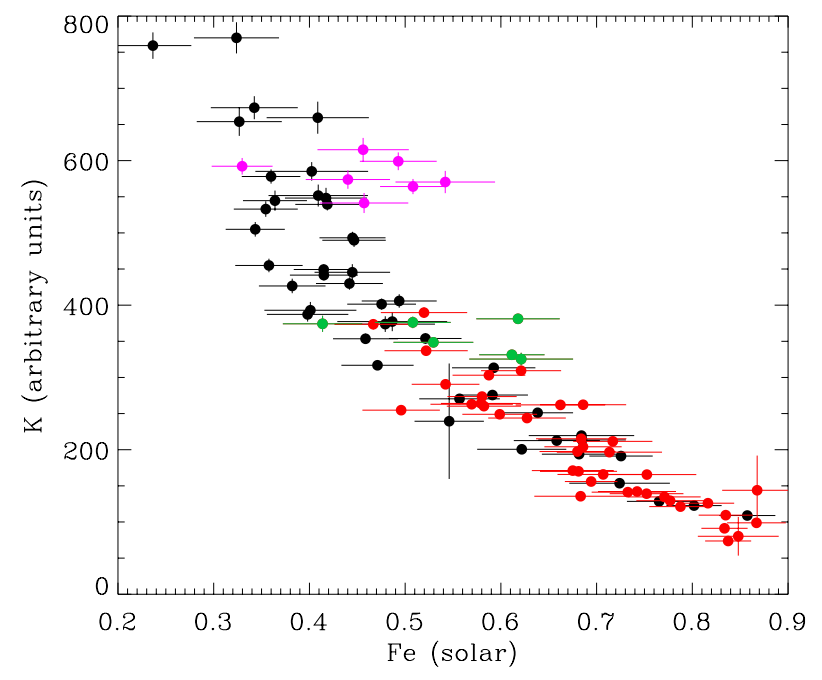

Fig. 15. K-Fe correlation. Red marks the IN, black the OUT polygons, magenta SE polygons OUT of the spiral, and green SE polygons IN the spiral. All the SE regions show a metal excess and introduce a significant scatter in the plot.

the same amount of $p \mathrm{~d} V$ work originally done by this gas will now be exerted on it. Net heating will be small, as it will be related to deviations from the adiabatic evolution of the sloshing gas, which, as discussed in Sect. 4.5, must be small.

\subsection{Southeast metal excess}

The metallicity map (see Fig. 9) shows a hint of a metallicity excess in the southeast (see also Ghizzardi et al. 2013).

In Fig. 15, we marked as magenta circles the southeast regions located outside the spiral. The plot confirms that these regions have a higher metal content, significantly higher than that of the regions with a similar entropy level. The nature of this excess cannot be immediately interpreted. The excess is located just outside the spiral pattern. This might suggest that some metals have been injected in the surrounding ICM. These regions are also close to the regions where Kelvin-Helmoltz instabilities might be at work (Sect. 4.3), which would facilitate the mixing of gas. We note, however, that the southeast regions located on the spiral show a similar metal excess. These regions are marked with green circles in Fig. 15. It is remarkable that all these regions (both magenta and green points) introduce a significant scatter in the K-Fe plot. Indeed, if we remove these points from the plot, the K-Fe correlation is significantly tighter. Although we lack a deep understanding of the origin of this metal excess, the fact that it involves both regions IN and OUT of the spiral leads us to think that it is most likely not due to mixing or diffusion processes. This is also supported by the fact that all the other regions of the cluster lie on the K-Fe correlation. A more attractive alternative is that this excess might trace some cluster asymmetry that predates the onset of the sloshing.

\section{Summary}

We analyzed two long XMM-Newton observations ( $120 \mathrm{ks})$ of A496. Taking advantage of the large collecting area and spectral resolution of XMM-Newton, the quality of the data allowed a detailed characterization of the metal abundance for this cluster. Our main results concern the detection of sloshing cold fronts and the connection between the metal distribution and the sloshing.

- We detected three and characterized two of the four cold fronts in A496. The main cold front is located in the NNW direction at a distance $\sim 60 \mathrm{kpc}$ from the X-ray peak. We also detected two cold fronts to the south $\sim 35$ and $\sim 160 \mathrm{kpc}$ from the center.

- We detected the low- temperature/entropy spiral feature found in many sloshing cores. All the cold fronts are located along the edge of the spiral; the southern outermost cold front is placed at the tail of the spiral.

- The Fe abundance drops abruptly across cold fronts. A similar but less significant trend is seen in Si and S.

- Our metallicity map shows that plasma in the spiral has a higher metal content than the surrounding medium.

- We found that regions within the spiral follow the same $\mathrm{K}-\mathrm{Fe}$ relation as regions outside the spiral.

All these results allowed us to assemble a fairly articulated picture of the evolution of the ICM in the core of A496:

- Using fiducial numbers for the age of the sloshing structure, we found that conduction between the gas in the spiral and the ambient medium must be suppressed by more than one order of magnitude with respect to Spitzer conductivity.

- Under the reasonable assumption that prior to the onset of sloshing the ICM was stratified according to entropy, we inferred that while the low-entropy metal-rich plasma is lifted up through the cluster atmosphere it suffers little or no heating/mixing with the ambient medium.

- While sloshing appears to be capable of lifting up significant amounts of gas, the limited heat exchange and mixing between gas within and outside the spiral implies that this mechanism is not at all effective in 1) permanently redistributing metals within the core region (the cooler metalricher gas will eventually fall back to the center) and 2) heating the coolest and densest gas, thereby providing little or no contribution to the staving off of catastrophic cooling in cool cores.

- The only indication we have that some mixing might (and we stress the might) be occurring comes from regions $\mathrm{E}$ and $\mathrm{W}$ of the center, where the spiral edges appear to be smeared out.

- Finally, within the quasi-adiabatic sloshing scenario we have outlined, the excess we observe both within and out of the spiral SE of the center most likely traces some cluster asymmetry that predates the onset of sloshing.

\section{References}

Akritas, M. G., \& Bershady, M. A. 1996, ApJ, 470, 706

Anders, E., \& Grevesse, N. 1989, Geochim. Cosmochim. Acta, 53, 197

Arnaud, K. A. 1996, in Astronomical Data Analysis Software and Systems V, eds. G. H. Jacoby, \& J. Barnes, ASP Conf. Ser., 101, 17

Asai, N., Fukuda, N., \& Matsumoto, R. 2005, Adv. Space Res., 36, 636

Asai, N., Fukuda, N., \& Matsumoto, R. 2007, ApJ, 663, 816

Ascasibar, Y., \& Markevitch, M. 2006, ApJ, 650, 102

Buote, D. A., Lewis, A. D., Brighenti, F., \& Mathews, W. G. 2003, ApJ, 594, 741

Canning, R. E. A., Sun, M., Sanders, J. S., et al. 2013, MNRAS, 435, 1108

Cappellari, M., \& Copin, Y. 2003, MNRAS, 342, 345

Carilli, C. L., \& Taylor, G. B. 2002, ARA\&A, 40, 319

Clarke, T. E., Blanton, E. L., \& Sarazin, C. L. 2004, ApJ, 616, 178

Clarke, T. E., Randall, S. W., Sarazin, C. L., Blanton, E. L., \& Giacintucci, S. 2013, ApJ, 772, 84

David, L. P., Jones, C., Forman, W., et al. 2009, ApJ, 705, 624 
De Grandi, S., \& Molendi, S. 2001, ApJ, 551, 153

De Grandi, S., \& Molendi, S. 2009, A\&A, 508, 565

De Grandi, S., Ettori, S., Longhetti, M., \& Molendi, S. 2004, A\&A, 419, 7

de Plaa, J., Werner, N., Simionescu, A., et al. 2010, A\&A, 523, A81

Diehl, S., \& Statler, T. S. 2006, MNRAS, 368, 497

Dupke, R., \& White, III, R. E. 2003, ApJ, 583, L13

Dupke, R., White, III, R. E., \& Bregman, J. N. 2007, ApJ, 671, 181

Dursi, L. J., \& Pfrommer, C. 2008, ApJ, 677, 993

Ettori, S. 2002, MNRAS, 330, 971

Ettori, S., \& Fabian, A. C. 2000, MNRAS, 317, L57

Ettori, S., Fabian, A. C., Allen, S. W., \& Johnstone, R. M. 2002, MNRAS, 331, 635

Ettori, S., Gastaldello, F., Gitti, M., et al. 2013, A\&A, 555, A93

Fabian, A. C., Sanders, J. S., Taylor, G. B., et al. 2006, MNRAS, 366, 417

Fabian, A. C., Sanders, J. S., Allen, S. W., et al. 2011, MNRAS, 418, 2154

Ferrari, C., Govoni, F., Schindler, S., Bykov, A. M., \& Rephaeli, Y. 2008,

Space Sci. Rev., 134, 93

Gaspari, M., \& Churazov, E. 2013, A\&A, 559, A78

Gaspari, M., Churazov, E., Nagai, D., Lau, E. T., \& Zhuravleva, I. 2014, A\&A, 569, A67

Gastaldello, F., Buote, D. A., Temi, P., et al. 2009, ApJ, 693, 43

Gastaldello, F., Di Gesu, L., Ghizzardi, S., et al. 2013, ApJ, 770, 56

Ghizzardi, S., Molendi, S., Pizzolato, F., \& De Grandi, S. 2004, ApJ, 609, 638

Ghizzardi, S., Rossetti, M., \& Molendi, S. 2010, A\&A, 516, A32

Ghizzardi, S., De Grandi, S., \& Molendi, S. 2013, Astron. Nachr., 334, 422

Giacintucci, S., Markevitch, M., Brunetti, G., Cassano, R., \& Venturi, T. 2011 A\&A, 525, L10

Giacintucci, S., Markevitch, M., Venturi, T., et al. 2014, ApJ, 781, 9

Govoni, F., Murgia, M., Markevitch, M., et al. 2009, A\&A, 499, 371

Graham, J., Fabian, A. C., Sanders, J. S., \& Morris, R. G. 2006, MNRAS, 368, 1369

Hallman, E. J., Skillman, S. W., Jeltema, T. E., et al. 2010, ApJ, 725, 1053

Kalberla, P. M. W., Burton, W. B., Hartmann, D., et al. 2005, A\&A, 440, 775

Laganá, T. F., Andrade-Santos, F., \& Lima Neto, G. B. 2010, A\&A, 511, A15

Lal, D. V., Kraft, R. P., Randall, S. W., et al. 2013, ApJ, 764, 83

Leccardi, A., \& Molendi, S. 2008, A\&A, 486, 359

Leccardi, A., Rossetti, M., \& Molendi, S. 2010, A\&A, 510, A82

Lyutikov, M. 2006, MNRAS, 373, 73

Ma, C.-J., Owers, M., Nulsen, P. E. J., et al. 2012, ApJ, 752, 139

Machacek, M. E., Jerius, D., Kraft, R., et al. 2011, ApJ, 743, 15

Markevitch, M., \& Vikhlinin, A. 2007, Phys. Rep., 443, 1

Markevitch, M., Ponman, T. J., Nulsen, P. E. J., et al. 2000, ApJ, 541, 542

Markevitch, M., Mazzotta, P., Vikhlinin, A., et al. 2003a, ApJ, 586, L19

Markevitch, M., Vikhlinin, A., \& Forman, W. R. 2003b, in ASP Conf. Ser. 301, eds. S. Bowyer, \& C.-Y. Hwang, 37

Mazzotta, P., \& Giacintucci, S. 2008, ApJ, 675, L9
Molendi, S., \& Gastaldello, F. 2009, A\&A, 493, 13

Nevalainen, J., David, L., \& Guainazzi, M. 2010, A\&A, 523, A22

O'Sullivan, E., David, L. P., \& Vrtilek, J. M. 2014, MNRAS, 437, 730

Owers, M. S., Nulsen, P. E. J., Couch, W. J., \& Markevitch, M. 2009a, ApJ, 704, 1349

Owers, M. S., Nulsen, P. E. J., Couch, W. J., Markevitch, M., \& Poole, G. B. 2009b, ApJ, 692, 702

Owers, M. S., Nulsen, P. E. J., \& Couch, W. J. 2011, ApJ, 741, 122

Paterno-Mahler, R., Blanton, E. L., Randall, S. W., \& Clarke, T. E. 2013, ApJ, 773,114

Peres, C. B., Fabian, A. C., Edge, A. C., et al. 1998, MNRAS, 298, 416

Ponman, T. J., Sanderson, A. J. R., \& Finoguenov, A. 2003, MNRAS, 343, 331

Randall, S. W., Jones, C., Kraft, R., Forman, W. R., \& O'Sullivan, E. 2009a, ApJ, 696,1431

Randall, S. W., Jones, C., Markevitch, M., et al. 2009b, ApJ, 700, 1404

Rasmussen, J., Ponman, T. J., \& Mulchaey, J. S. 2006, MNRAS, 370, 453

Rebusco, P., Churazov, E., Böhringer, H., \& Forman, W. 2006, MNRAS, 372, 1840

Roediger, E., \& ZuHone, J. A. 2012, MNRAS, 419, 1338

Roediger, E., Lovisari, L., Dupke, R., et al. 2012, MNRAS, 420, 3632

Roediger, E., Kraft, R. P., Forman, W. R., Nulsen, P. E. J., \& Churazov, E. 2013, ApJ, 764, 60

Rossetti, M., Eckert, D., De Grandi, S., et al. 2013, A\&A, 556, A44

Rossetti, M., Ghizzardi, S., Molendi, S., \& Finoguenov, A. 2007, A\&A, 463, 839

Sanders, J. S., \& Fabian, A. C. 2006, MNRAS, 371, 1483

Sanders, J. S., Fabian, A. C., \& Taylor, G. B. 2005, MNRAS, 356, 1022

Sanders, J. S., Fabian, A. C., \& Taylor, G. B. 2009, MNRAS, 393, 71

Simionescu, A., Werner, N., Forman, W. R., et al. 2010, MNRAS, 405, 91

Simionescu, A., Werner, N., Urban, O., et al. 2012, ApJ, 757, 182

Spitzer, L. 1962, Physics of Fully Ionized Gases (New York: Interscience publishers)

Tanaka, T., Kunieda, H., Hudaverdi, M., Furuzawa, A., \& Tawara, Y. 2006, PASJ, 58,703

Tittley, E. R., \& Henriksen, M. 2005, ApJ, 618, 227

Vazza, F., Roediger, E., \& Brüggen, M. 2012, A\&A, 544, A103

Vikhlinin, A., Markevitch, M., \& Murray, S. S. 2001a, ApJ, 551, 160

Vikhlinin, A., Markevitch, M., \& Murray, S. S. 2001b, ApJ, 549, L47

Vikhlinin, A. A., \& Markevitch, M. L. 2002, Astron. Lett., 28, 495

Voit, G. M., Kay, S. T., \& Bryan, G. L. 2005, MNRAS, 364, 909

ZuHone, J. A., Markevitch, M., \& Johnson, R. E. 2010, ApJ, 717, 908

ZuHone, J. A., Markevitch, M., \& Lee, D. 2011, ApJ, 743, 16

ZuHone, J. A., Markevitch, M., Brunetti, G., \& Giacintucci, S. 2013a, ApJ, 762, 78

ZuHone, J. A., Markevitch, M., Ruszkowski, M., \& Lee, D. 2013b, ApJ, 762, 69

Pages 14 to 16 are available in the electronic edition of the journal at http://wWw. aanda.org 


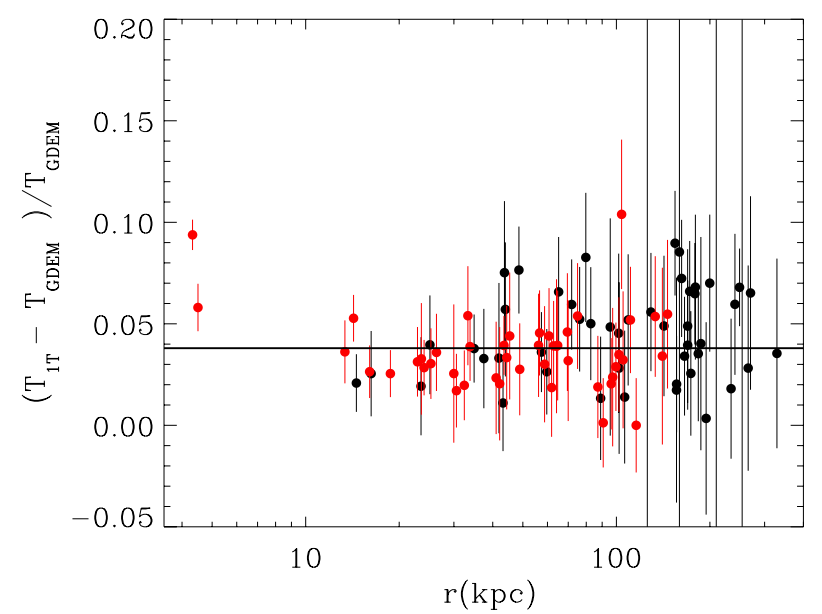

Fig. A.1. Relative temperature differences between the $1 \mathrm{~T}$ and GDEM models as a function of the distance from the center of the cluster. Red marks the IN, black the OUT polygons.

\section{Appendix A: Comparison of spectral models}

We have performed a detailed analysis of the temperature structure of the regions of interest in A496 (annular sectors and polygons), searching for possible multitemperature components in the ICM spectra.

In this Appendix we present the results from the analysis of each spectrum with the two different spectral models described in Sect. 2.2: the one-temperature vapec model (1T model), and the multitemperature GDEM model (Buote et al. 2003).

In Fig. A.1 we show the relative differences between the temperatures estimated with the $1 \mathrm{~T}$ model, $T_{1 T}$, and the GDEM model, $T_{\mathrm{GDEM}}$, that is $\left(T_{1 T}-T_{\mathrm{GDEM}}\right) / T_{\mathrm{GDEM}}$, plotted as a function of the physical distance from the cluster center of the regions for the 98 polygons used to extract the spectra (see Sect. 3.4 and Fig. 8). In Fig. A.1 only the central bin shows a temperature difference significantly larger than $\sim 5 \%$, while in all other bins the differences are around 3-6\% and consistent with this value within $1-2 \sigma$. The best-fitting constant model, with the exclusion of the first bin, gives $0.038 \pm 0.002$ and is plotted in Fig. A.1 as a solid line.

The relative $\mathrm{Fe}$ abundance differences, $\left(Z_{\mathrm{Fe}, 1 \mathrm{~T}}-\right.$ $\left.Z_{\mathrm{Fe}, \mathrm{GDEM}}\right) / Z_{\mathrm{Fe}, \mathrm{GDEM}}$, give similar results (see Fig. A.2). In this case, the best-fitting constant model gives $0.035 \pm 0.01$.

We can ascribe the small $\sim 3 \%$ bias toward slightly higher temperatures and $\mathrm{Fe}$ abundances from the $1 \mathrm{~T}$ model to wellknown calibration problems in the EPIC detector between the soft $(0.7-2 \mathrm{keV})$ and hard (2.-8. keV) X-ray bands (e.g., Nevalainen et al. 2010).

From the negligible differences between temperatures and $\mathrm{Fe}$ abundances in the two spectral models it is clear that, if present, a multitemperature component in the ICM contributes only modestly to the total emission. More interestingly for the purposes of our work, the value of the measured $\mathrm{Fe}$ abundance is essentially unaffected by a possible multitemperature structure.

We excluded from our analysis the central bin, which is the only one where the relative differences are significant in both cases. This central cluster region might be contaminated by the central AGN or by a true multiphaseness of the ICM.

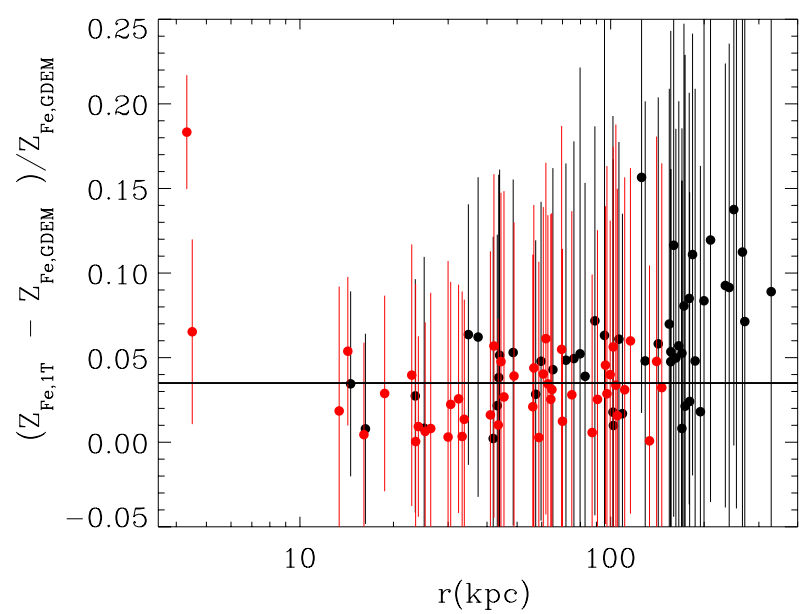

Fig. A.2. Relative $\mathrm{Fe}$ abundance differences between the $1 \mathrm{~T}$ and GDEM models as a function of the distance from the center of the cluster. Red marks the IN, black the OUT polygons.

\section{Appendix B: Pressure jump across the cold fronts}

In Sect. 3.2 we derived the pressure jump across the main cold fronts: NNW, divided into two sectors $30^{\circ}-75^{\circ}$ and $75^{\circ}-120^{\circ}$ to account for its boxy morphology, and $\mathrm{S} 2$.

To a first approximation, we derived the pressure jump across the front by using the electron density and the temperature in the two bins just inside and outside each edge. Namely, we modeled the electron density profile with a broken power law:

$n= \begin{cases}n_{\text {in }}\left(\frac{r}{r_{\mathrm{CF}}}\right)^{-\alpha_{\text {in }}} & r<r_{\mathrm{CF}} \\ n_{\mathrm{out}}\left(\frac{r}{r_{\mathrm{CF}}}\right)^{-\alpha_{\mathrm{out}}} & r>r_{\mathrm{CF}},\end{cases}$

where $n_{\text {in }}$ and $n_{\text {out }}$ are the electron densities at the cold-front position $r_{\mathrm{CF}}$ on the inner and outer side of the edge. We projected the emissivity along the line of sight and fit the surface brightness profiles of each sector of interest to derive the values of $n_{\text {in }}, n_{\text {out }}, \alpha_{\text {in }}$, and $\alpha_{\text {out }}$. The fitting process was divided into two steps: we initially fit only the outer part of the profile (outside the cold front) where only the external component is present and derived the $n_{\text {out }}$ and $\alpha_{\text {out }}$ parameters. Then, we fixed the external component and fit the whole profile to derive the inner component parameters. Finally, we derived the pressure jump $p_{\text {in }} / p_{\text {out }}=n_{\text {in }} T_{\text {in }} / n_{\text {out }} T_{\text {out }}$ where the temperature values $T_{\text {in }}$ and $T_{\text {out }}$ in the two bins close to the front position were obtained through the spectral analysis (see Fig. 7). The pressure jumps obtained through this method are reported in the second column (approx pressure jump) of Table B.1.

A more accurate estimate for the pressure profile can be obtained by multiplying the electron density profile with the temperature profile. However, the temperature profiles (see Fig. 7) are evaluated in bins that are significantly larger than the bins available for surface brightness (and electron density) profiles. We then interpolated the temperature profiles to reconstruct a temperature profile from the same bins that were used for the density profile. We performed two independent interpolations for the points inside and outside the cold front. For the outer regions we excluded the points close to the cold front to keep far away from the stagnation point; profiles in this region were computed by extrapolating measures at larger radii. We note that since bins used for the temperature profiles are large to allow reliable spectrum fitting and the number of bins is quite small, we did not deproject the temperature. 

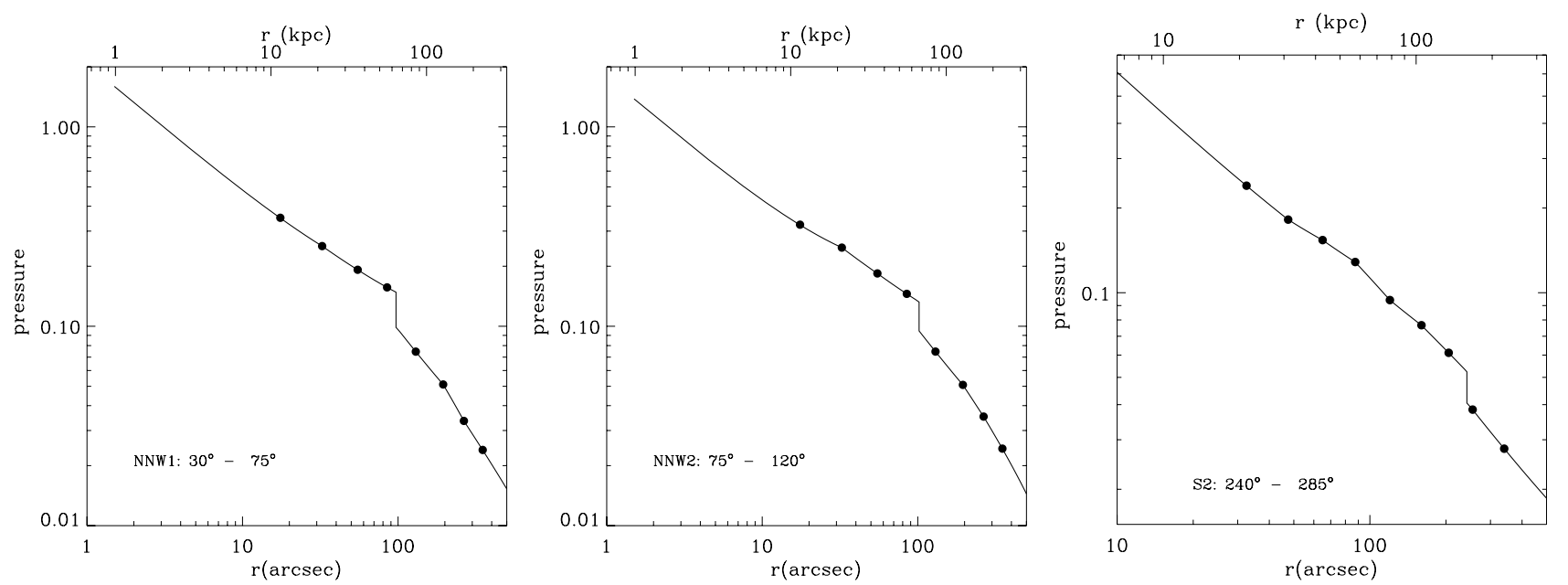

Fig. B.1. Pressure (arbitrary units) profiles obtained with the two different methods (see text) in the three cold-front sectors $30^{\circ}-75^{\circ}$ (left panel), $75^{\circ}-120^{\circ}$ (middle panel) and $240^{\circ}-285^{\circ}$ (right panel). The solid line is the pressure profile obtained by using the interpolated temperature profile, filled black circles correspond to the pressure obtained by using the original $T$ profile.

Table B.1. Pressure jumps for the three cold-front sectors

\begin{tabular}{lcc}
\hline \hline Sector & Approx pressure jump & Pressure jump \\
\hline NNW: $30^{\circ}-75^{\circ}$ & $1.40 \pm 0.03$ & $1.5 \pm 0.05$ \\
NNW: $75^{\circ}-120^{\circ}$ & $1.33 \pm 0.02$ & $1.40 \pm 0.05$ \\
S2: $240^{\circ}-285^{\circ}$ & $1.28 \pm 0.03$ & $1.29 \pm 0.05$ \\
\hline
\end{tabular}

In Fig. B.1 we plot the pressure profiles (solid line) derived for the three cold-front sectors. The corresponding pressure jumps are reported in the last column of Table B.1. As expected, the jumps measured from the pressure profiles are slightly larger than the approximated ones: $\sim 7 \%$ and $\sim 5 \%$ for the two NNW sectors and $0.7 \%$ for $\mathrm{S} 2$.

In Fig. B. 1 we also overplot the $n T$ profiles obtained from the original temperature profiles. The figure confirms that the two profiles match and that the differences between the results obtained with the two procedures are small.

\section{Appendix C: Testing the robustness of the K-Fe correlation}

In this appendix we address the robustness of the correlation $(\mathrm{K}-\mathrm{Fe})$ between entropy and metal abundance. We wish to investigate whether the match between IN and OUT regions in the K-Fe plot might be due to the single-temperature model employed to fit the spectra. If, in the regions on the spiral there were some gas mixing, two different gas phases would coexist and the $1 \mathrm{~T}$ fitting might provide an averaged value for $T, Z, n_{\mathrm{e}}$ which could preserve the correlation even if the gas were made up of two distinct components. Moreover, even if the gas whitin the spiral is not affected by mixing, projection effects can lead to the same bias since the analyzed spectrum would contain the two components. We fitted the spectra with a two-temperature model, but using this model did not improve the quality of the fit. We therefore took a different approach: we built a composite spectrum that contained the spectra of two different regions at opposite sides in the $\mathrm{K}-\mathrm{Fe}$ plot. This simulates both a region containing mixed gas and a region affected by projection.

We chose regions \#2 and \#71 (see Fig. C.1). The lowest temperature region (\#2) is on the spiral feature at the center of the cluster, while region \#71 is outside the spiral north $\sim 200^{\prime \prime}$ $(\sim 120 \mathrm{kpc})$ of the center.

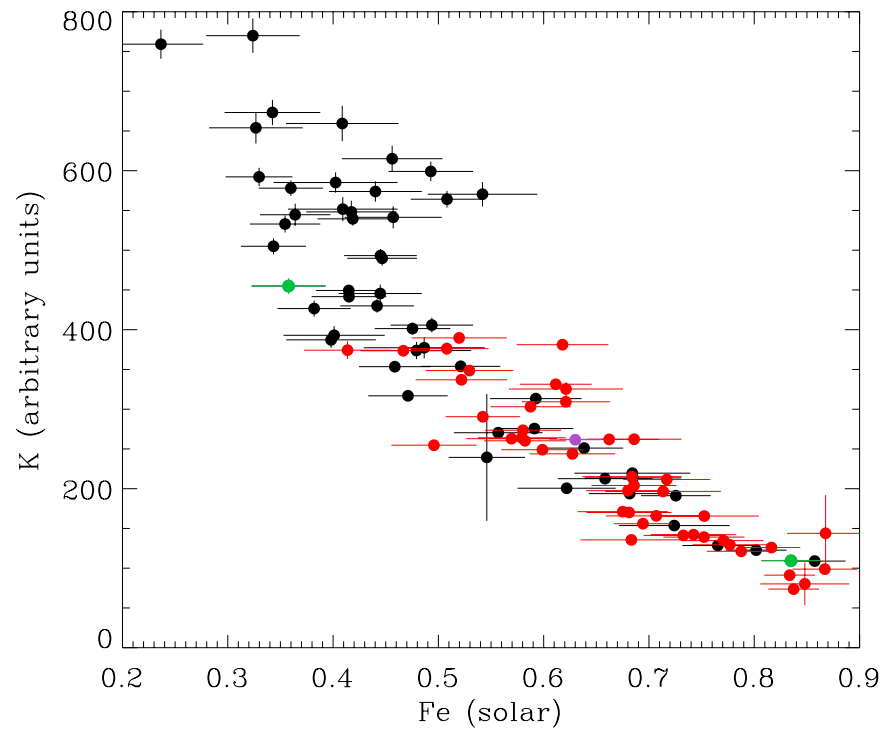

Fig. C.1. K-Fe correlation. Red marks the IN, black the OUT polygons. Green points mark regions \#2 and \#71 used for the test, the purple point represents the composite region $\# 2+\# 71$.

We checked the count rates of both regions \#2 and \#71 and verified that they have similar values, so that the two regions contribute at the same level to the total spectrum. This mimics a region where mixing occurs with similar filling factors for the two different gas phases. We first fit the composite spectrum with a 1T model and found, as expected, best-fit values of the temperature and of the metal abundance (see Table C.1) that are in between those measured for regions \#2 and \#71. This also leads to a point in the K-Fe plot that is indeed still on the relation (see Fig. C.1), but in this case, the fitting procedure returns a much higher $\chi^{2}, 760.3$ for 676 d.o.f. against $\chi^{2}=576.2$ for 455 d.o.f. for region $\# 2$ and $\chi^{2}=520.5$ for 540 d.o.f. for region \#71. Furthermore, visual inspection of the residuals clearly indicates that there is more than one spectral component. We then fit the composite spectrum with a $2 \mathrm{~T}$ model, leaving normalization, temperature and Fe abundance as free parameters for both components. This resulted in a highly significant improvement in the fit ( $\chi^{2}$ of 652.5 vs. 673 d.o.f.) and in the disappearance of the 
Table C.1. Best-fit values for temperature and metal abundance obtained using the $1 \mathrm{~T}$ and $2 \mathrm{~T}$ model for regions \#2, \#71 and for the composite spectrum \#2+\#71.

\begin{tabular}{|c|c|c|c|c|c|c|c|c|c|c|}
\hline & \multicolumn{3}{|c|}{$1 \mathrm{~T}$} & \multicolumn{5}{|c|}{$2 \mathrm{~T}$} & \multirow[t]{2}{*}{$\mathrm{F}$} & \multirow[t]{2}{*}{$\mathrm{P}$} \\
\hline & $T(\mathrm{keV})$ & $Z_{\mathrm{Fe}}($ solar $)$ & $\chi^{2} /$ d.o.f. & $T_{1}(\mathrm{keV})$ & $Z_{1, \mathrm{Fe}}$ (solar) & $T_{2}(\mathrm{keV})$ & $Z_{2, \mathrm{Fe}}($ solar $)$ & $\chi^{2} /$ d.o.f. & & \\
\hline$\# 2$ & $2.58 \pm 0.02$ & $0.83 \pm 0.04$ & $576.2 / 455$ & $1.72_{-0.05}^{+0.24}$ & unconstrained & $2.89 \pm 0.4$ & $0.57_{-0.11}^{+0.06}$ & $543.0 / 452$ & 9.21 & $6.33 \times 10^{-6}$ \\
\hline \#71 & $4.80 \pm 0.10$ & $0.34 \pm 0.04$ & $520.5 / 540$ & $2.39_{-0.43}^{+1.03}$ & $0.13_{-0.06}^{+0.10}$ & $6.85_{-0.35}^{+2.14}$ & $0.48_{-0.09}^{+0.32}$ & $508.9 / 537$ & 4.09 & $6.93 \times 10^{-3}$ \\
\hline$\# 2+\# 71$ & $3.29 \pm 0.03$ & $0.61 \pm 0.01$ & $760.3 / 676$ & $2.27 \pm 0.13$ & $0.55 \pm 0.05$ & $6.84 \pm 0.7$ & $0.49 \pm 0.07$ & $652.5 / 673$ & 63.27 & $4.76 \times 10^{-36}$ \\
\hline
\end{tabular}

Notes. The two last columns report the F-test value and the corresponding probability.

structures in the residuals found in the $1 \mathrm{~T}$ fit. We performed the same $2 \mathrm{~T}$ fitting also on the spectra extracted from regions \#2 and \#71. Here, including a second component provided improvements that are significantly smaller than those found for the composite region (see Table C.1). These improvements, while formally statistically significant, as indicated by the results of the F-test (see Table C.1), can be ascribed to the known EPIC calibration mismatch between soft and hard spectral bands (e.g., Nevalainen et al. 2010).
We conducted the same analysis on other pairs of spectra and found similar results.

We conclude that while mixing of different phases can lead to spectra whose $1 \mathrm{~T}$ best fits lie on the K-Fe correlation, such composite spectra would be easily identified with a multitemperature analysis. The lack of any evidence of multiphaseness, beyond the modest level expected from the known EPIC calibration issues, tells us that mixing must at the most be modest in the gas located within the spiral. 